Geometry $\&$ Topology

Volume 9 (2005) 1835-1880

Published: 25 September 2005

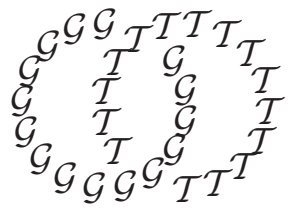

\title{
The Grushko decomposition of a finite graph of finite rank free groups: an algorithm
}

\author{
GuO-An Diao \\ MARK FEIGHN \\ School of Arts and Sciences, Holy Family University \\ Philadelphia, PA 19114, USA \\ and \\ Department of Mathematics and Computer Science \\ Rutgers University, Newark, NJ 07102, USA
}

Email: gdiao@holyfamily.edu and feighn@andromeda.rutgers.edu

\begin{abstract}
A finitely generated group admits a decomposition, called its Grushko decomposition, into a free product of freely indecomposable groups. There is an algorithm to construct the Grushko decomposition of a finite graph of finite rank free groups. In particular, it is possible to decide if such a group is free.
\end{abstract}

\section{AMS Classification numbers Primary: 20F65}

Secondary: 20E05

Keywords: Graph of groups, Grushko decomposition, algorithm, labeled graph

Proposed: Benson Farb

Seconded: Martin Bridson, Joan Birman
Received: 5 February 2005

Revised: 11 September 2005 


\section{Introduction}

Theorem 1.1 (Grushko [15]) A finitely generated group $G$ is a free product of a finite rank free subgroup and finitely many freely indecomposable non-free subgroups.

Up to reordering and conjugation, the non-free factors appearing in this the Grushko decomposition of $G$ are unique. The rank of the free factor is also an invariant of $G$. The main result of this paper is:

Theorem 1.2 There is an algorithm which produces the Grushko decomposition of a finite graph 1 of finite rank free group 4 .

A relative version is given in Section 2.11

The class of finite graphs of finite rank free groups is fascinating and has received much attention. For example, mapping tori of free group automorphisms are in this class, see [3, 10, 12, 7]. Also, limit groups which appear in the recent work on the Tarski problem, see [19, 24, have a hierarchy in which those limit groups appearing on the first level are finite graphs of finite rank free groups.

The algorithm is given in Section 2. Theorem 2.8 is a refined version of Theorem 1.2 and is proved in Section 10. Work of Shenitzer or Swarup when combined with Whitehead's algorithm for deciding if a given element of a free group is primitive 3 gives the case of Theorem 1.2 where edge groups are cyclic.

Theorem 1.3 (Shenitzer-Swarup [26, 29, 31, 30], see also [28]) There is an algorithm to decide whether a finite graph of finite rank free groups with cyclic edge groups is free.

There are other notable situations where the Grushko decomposition may be found algorithmically. For example, given a presentation with one relation for a group $G$, the Grushko decomposition of $G$ can be constructed (eg, 21]). Also, from a triangulation of a closed orientable 3-manifold $M$, the connected sum decomposition of $M$ can be found (see [17). The referee points out two other papers [13] and 20] that consider respectively hyperbolic groups and limit groups.

\footnotetext{
${ }^{1}$ in the sense of Bass-Serre 25

${ }^{2}$ ie, vertex and edge groups are free of finite rank, see Section 2.7

${ }^{3}$ an element of some basis
} 
Here is a sketch of the proof of Theorem 1.2. There are three steps. Suppose that $S$ is a cocompact $G$-tree with finitely generated edge stabilizers. Suppose further that $G$ is freely decomposable and that $T$ is a $G$-tree with one orbit of edges and with trivial edge stabilizers. Give the product $S \times T$ the diagonal $G$-action. $S \times T$ is a union of squares (edge $\times$ edge). There is a cocompact $G-$ subcomplex $X_{S}(T)$ that is a $G$-deformation retract of $S \times T$ (see Section 5 ). As $X_{S}(T)$ is contained in $S \times T$, there is a natural map $X_{S}(T) \rightarrow T$. The preimage in $X_{S}(T)$ of the midpoint of an edge of $T$ is a compact forest. A valence one vertex of this forest corresponds to a square in $X_{S}(T)$ that may be equivariantly collapsed. We may iteratively collapse until each component of this tree is a point. These points exhibit free decompositions of $G$ that are compatible with the original splitting given by $S$.

An argument similar to the one in this first step was used by Bestvina-Feighn in 2 to among other things reprove Theorem 1.3. The use of products of trees is inspired by the Fujiwara-Papasoglu [1] approach to the Rips-Sela JSJtheorem [23].

The second step is to translate these collapses of $X_{S}(T)$ into corresponding simplifications of the original graph of groups. This is straightforward and is done in Section 8 . These first two steps do not use the hypothesis that edge and vertex groups are free.

In the third step (Section 9), we show how Gersten representatives [14] of conjugacy classes of subgroups of free groups can be used to detect simplifications. This is probably the heart of the paper.

A special case of Theorem 1.2 solves Problem F24b on the problem list at http://www.grouptheory.org.

For the convenience of readers interested primarily in using the algorithm, it is described in the next section (Section 2). Definitions are given, but proofs are, for the most part, deferred until later in the paper. The first two steps of the proof are more general and therefore somewhat cleaner, see Sections 3 , 8 wich can be read independently of Section 2 .

The first named author's thesis [9] included an algorithm to decide if a finite graph of finite rank free groups is free. The second named author warmly thanks Mladen Bestvina for helpful conversations and gratefully acknowledges the support of the National Science Foundation. 


\section{The algorithm}

Mainly to establish notation, we first recall the definition of a graph of groups.

\section{$2.1 \quad$ Graphs of groups}

A reference for this section is 25. A graph is a 1-dimensional $C W$-complex and is determined by the following combinatorial data: a 4 -tuple $\left(V, \hat{E}\right.$, op, $\left.\partial_{0}\right)$ where

- $V$ and $\hat{E}$ are sets;

- op: $\hat{E} \rightarrow \hat{E}$ satisfies

(1) op $\circ$ op $=\mathrm{Id}$, and

(2) $\operatorname{op}(e) \neq e$, for all $e \in \hat{E}$; and

- $\partial_{0}: \hat{E} \rightarrow V$.

For $e \in \hat{E}$, we also write $e^{-1}$ for $\mathrm{op}(e)$ and set $\partial_{1} e=\partial_{0} e^{-1}$. For $v \in V$, $\hat{E}(v)=\left\{e \in \hat{E}: \partial_{0} e=v\right\}$. The valence of $v$ is the cardinality $|\hat{E}(v)|$ of $\hat{E}(v)$. Such a 4 -tuple is a combinatorial graph. The graph

$$
\Gamma=\Gamma\left(V, \hat{E}, \text { op, } \partial_{0}\right)
$$

so determined has vertex set identified with $V$. The set $\hat{E}$ corresponds to the set of oriented edges of $\Gamma$; the set $E$ of edges of $\Gamma$ is identified with $\left\{\left\{e, e^{-1}\right\}: e \in \hat{E}\right\}$. The map op reverses edge orientations, and $\partial_{0}$ determines the characteristic maps of $\Gamma$. Up to isomorphism, a graph uniquely determines a combinatorial graph and vice versa. In particular, properties of one give properties of the other. The interior of an edge $e$ is denoted $\stackrel{e}{\text {. }}$

A graph of groups is a 4-tuple

$$
\mathcal{G}=\left(\Gamma\left(V, \hat{E}, \text { op, } \partial_{0}\right),\left\{G_{v}: v \in V\right\},\left\{G_{e}: e \in \hat{E}\right\},\left\{\varphi_{e}: e \in \hat{E}\right\}\right)
$$

where

- $\Gamma\left(V, \hat{E}\right.$, op, $\left.\partial_{0}\right)$ is a connected combinatorial graph $\Gamma(\mathcal{G})$;

- for $e \in \hat{E}$ and $v \in V, G_{e}=G_{e^{-1}}$ and $G_{v}$ are groups; and

- for $e \in \hat{E}, \varphi_{e}: G_{e} \rightarrow G_{\partial_{0} e}$ is a monomorphism.

The groups $G_{e}$ and $G_{v}$ are respectively edge and vertex groups. The $\varphi_{e}$ 's are bonding maps. We say that $\mathcal{G}$ is reduced if

- $\varphi_{e}: G_{e} \rightarrow G_{v}$ is not an isomorphism for any valence one vertex $v$; and 
- if $v$ has valence two and if $\varphi_{e}: G_{e} \rightarrow G_{v}$ is an isomorphism, then $\hat{E}(v)=$ $\left\{e, e^{-1}\right\}$ (in which case $\Gamma(\mathcal{G})$ is a loop).

Associated to a graph of groups $\mathcal{G}$ is an isomorphism type of group $\pi_{1}(\mathcal{G})$, see [25]. If $G \cong \pi_{1}(\mathcal{G})$ then we say that $\mathcal{G}$ is a graph of groups decomposition for $G$. Let $\mathcal{G}$ and $\mathcal{G}^{\prime}$ be graphs of groups with the same underlying graphs, edge groups, and vertex groups. We say that $\mathcal{G}$ and $\mathcal{G}^{\prime}$ are conjugate, written $\mathcal{G} \sim \mathcal{G}^{\prime}$, if there is a sequence $\vec{h}=\left\{h_{e} \in G_{\partial_{0} e}: e \in \hat{E}\right\}$ such that $\varphi_{e}^{\prime}=i_{h_{e}} \circ \varphi_{e}$ where $i_{h_{e}}$ denotes the inner automorphism induced by $h_{e}$, ie, $i_{h_{e}}(g)=h_{e} g h_{e}^{-1}$. If $\mathcal{G}$ and $\mathcal{G}^{\prime}$ are conjugate, then $\pi_{1}\left(\mathcal{G}^{\prime}\right)$ and $\pi_{1}(\mathcal{G})$ are isomorphic.

A simplicial action of a group $G$ on a tree $T$ determines a graph of groups with underlying graph $T / G$ and with vertex and edge groups given by vertex and edge stabilizers of in $T$. Conversely, a graph of groups $\mathcal{G}$ determines up to simplicial isomorphism a $G \cong \pi_{1}(\mathcal{G})$-tree $T(\mathcal{G})$. See [25. $\mathcal{G}$ is a trivial graph of groups decomposition if $G \cong \pi_{1}(\mathcal{G})$ fixes a point of $T(\mathcal{G})$. If $\mathcal{G}$ is a non-trivial graph of groups decomposition for $G$ then we also say that $\mathcal{G}$ is a splitting for $G$. If the edge groups of $\mathcal{G}$ are contained in some class of groups then we say that $G$ splits over this class. For example, if $\mathcal{G}$ is a non-trivial graph of groups decomposition for $G$ and all edge groups of $\mathcal{G}$ are trivial then we say that $G$ splits over $\mathbf{1}$ where $\mathbf{1}$ denotes the trivial group.

If $\mathcal{G}$ is a graph of groups with $G \cong \pi_{1}(\mathcal{G})$ then the edges $e$ of $\Gamma(\mathcal{G})$ with $G_{e}=\mathbf{1}$ determine a free product decomposition $F_{m} * G_{1} * \cdots * G_{n}$ where $m$ is the rank of the graph obtained from $\Gamma(\mathcal{G})$ by collapsing all edges $f$ with $G_{f} \neq \mathbf{1}$ and the $G_{i}$ 's are the fundamental groups of graphs of groups given by the components of $\Gamma(\mathcal{G}) \backslash\left(\cup_{e}\left\{\stackrel{e}{\mid} G_{e}=\mathbf{1}\right\}\right)$. This decomposition is called the decomposition of $G$ determined by the edges of $\mathcal{G}$ with trivial stabilizer.

Now we describe some operations on a graph of groups $\mathcal{G}$. These will be the simplifying moves of the algorithm. The moves will transform

$$
\mathcal{G}=\left(\Gamma,\left\{G_{v}\right\},\left\{G_{e}\right\},\left\{\varphi_{e}\right\}\right)
$$

into $\mathcal{G}^{\prime}=\left(\Gamma^{\prime},\left\{G_{v^{\prime}}\right\},\left\{G_{e^{\prime}}\right\},\left\{\varphi_{e^{\prime}}\right\}\right)$. Much of the data will be the same for $\mathcal{G}$ and $\mathcal{G}^{\prime}$, so in describing the moves we will usually only record the differences.

\section{$2.2 \quad$ Reducing}

If a bonding map at a valence one or two vertex is an isomorphism then there is an obvious simplification that we now describe.

Suppose $v \in V$ has valence one, ie, $\hat{E}(v)=\{e\}$. Suppose further that $\varphi_{e}$ is an isomorphism. Then, define $\mathcal{G}^{\prime}$ by setting $V^{\prime}=V \backslash\{v\}$ and $\hat{E}^{\prime}=\hat{E} \backslash\left\{e, e^{-1}\right\}$. 
Next suppose that $v \in V$ has valence two and that $\Gamma$ is not a loop, ie, $\hat{E}(v)=$ $\left\{e, f \neq e^{-1}\right\}$. Suppose further that $\varphi_{e}$ is an isomorphism. Then, define $\mathcal{G}^{\prime}$ by setting $V^{\prime}=V \backslash\{v\}$, setting $\hat{E}^{\prime}=\hat{E} \backslash\left\{e, e^{-1}\right\}$, redefining $\partial_{0} f$ to be $\partial_{1} e$, and redefining $\varphi_{f}$ to be $\varphi_{e^{-1}} \circ \varphi_{e}^{-1} \circ \varphi_{f}$.

If $\Gamma(\mathcal{G})$ is finite then, since each of these operations decreases the number of vertices, after finitely many operations we obtain a reduced graph of groups that has been obtained from $\mathcal{G}$ by reducing. See Figure 1 .

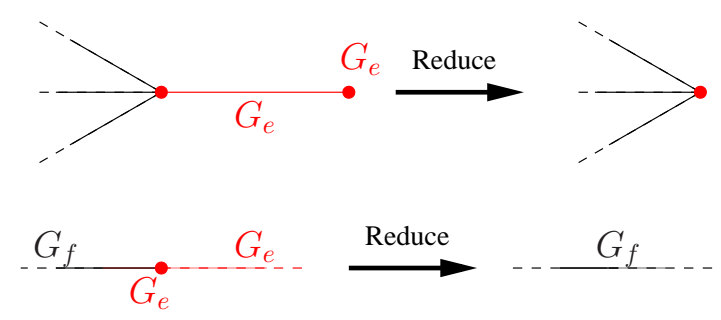

Figure 1: Reducing

In the remaining moves, some vertex group admits a graph of groups decomposition that is compatible with the bonding maps.

\section{$2.3 \quad$ Blowing up}

There are two types of blowing up. Suppose first that, for some $v \in V, G_{v}=$ $G_{v}^{\prime} *\langle t\rangle$ where $t$ has infinite order, and $\varphi_{e}\left(G_{e}\right) \subset G_{v}^{\prime}$ for $e \in \hat{E}(v)$. Then, define $\mathcal{G}^{\prime}$ as follows:

- the vertex sets are the same, ie, $V^{\prime}=V$;

- add a new oriented loop so that $\hat{E}^{\prime}=\hat{E} \cup\left\{e_{t}, e_{t}^{-1}\right\}$, with $\partial_{0} e_{t}=\partial_{0} e_{t}^{-1}=$ $v, G_{e_{t}}=\mathbf{1}$, and redefine $G_{v}$ to be $G_{v}^{\prime}$; and

- bonding maps are given by restricting the codomains of the bonding maps of $\mathcal{G}^{\prime}$ if necessary.

Secondly, suppose that, for some $v \in V, G_{v}=G_{v}^{\prime} * G_{v}^{\prime \prime}$ and, for each $e \in \hat{E}(v)$, $\varphi_{e}\left(G_{e}\right)$ is either contained in $G_{v}^{\prime}$ or $G_{v}^{\prime \prime}$. Then, define $\mathcal{G}^{\prime}$ as follows:

- replace $v$ by two vertices $v^{\prime}$ and $v^{\prime \prime}$, ie, $V^{\prime}=V \cup\left\{v^{\prime}, v^{\prime \prime}\right\} \backslash\{v\}$;

- add a new oriented edge so that $\hat{E}^{\prime}=\hat{E} \cup\left\{e_{t}, e_{t}^{-1}\right\}$ with $\partial_{0} e_{t}=v^{\prime}$, $\partial_{1} e_{t}=v^{\prime \prime}$, and $G_{e_{t}}=\mathbf{1}$ 


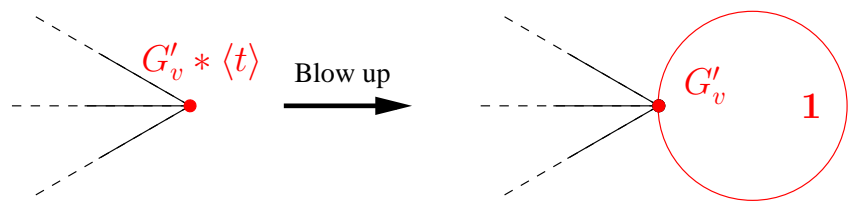

Figure 2: The first type of blowup

- if $e \in \hat{E}(v)$ then $\partial_{0} e$ is $v^{\prime}$ or $v^{\prime \prime}$ depending on whether $G_{e} \subset G_{v^{\prime}}$ or $G_{e} \subset G_{v^{\prime \prime}} ;$ and

- bonding maps are given by restricting the codomains of the bonding maps of $\mathcal{G}^{\prime}$ if necessary.

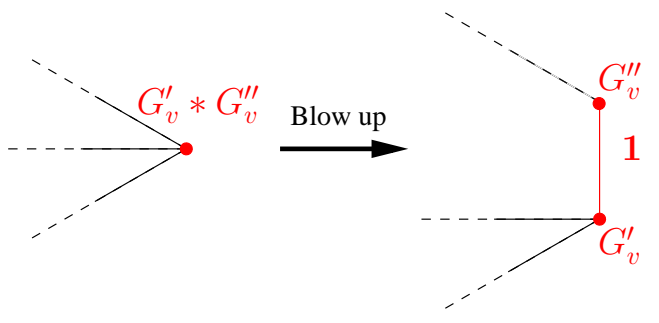

Figure 3: The second type of blowup

In each of these cases, we say that the new graph of groups is obtained from $\mathcal{G}$ by blowing up. See Figures 2 and 3 .

\subsection{Unpulling}

Suppose that, for some $v \in V$ and $e \in \hat{E}(v)$, we have $G_{v}=G_{v}^{\prime} * \mathbb{Z}, G_{e}=G_{e}^{\prime} * \mathbb{Z}$, $\varphi_{e}\left(G_{e}^{\prime}\right) \subset G_{v}^{\prime}, \varphi_{e}(\mathbb{Z})=\mathbb{Z}$, and $\varphi_{f}\left(G_{f}\right) \subset G_{v}^{\prime}$ for $f \in \hat{E}(v) \backslash\{e\}$. Then, define $\mathcal{G}^{\prime}$ as follows:

- $V^{\prime}=V$;

- $\hat{E}^{\prime}=\hat{E}$;

- redefine $G_{v}$ to be $G_{v}^{\prime}, G_{e}$ to be $G_{e}^{\prime}$; and

- bonding maps are given by restricting codomains of bonding maps of $\mathcal{G}^{\prime}$ if necessary.

We say that the new graph of groups is obtained from $\mathcal{G}$ by unpulling 4 See Figure 4

\footnotetext{
${ }^{4}$ We use the term unpulling because the inverse operation pulls an element of $G_{v}^{\prime}$ across the edge e.
} 


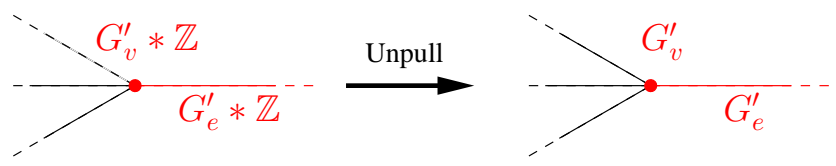

Figure 4: Unpulling

\subsection{Unkilling}

Suppose that, for some $v \in V$ and $e \in \hat{E}(v), G_{v}=G_{v}^{\prime} *\langle t\rangle$ where $t$ has infinite order, $G_{e}=G_{e}^{\prime} * G_{e}^{\prime \prime}, \varphi_{e}\left(G_{e}^{\prime}\right) \subset G_{v}^{\prime}, \varphi_{e}\left(G_{e}^{\prime \prime}\right) \subset t G_{v}^{\prime} t^{-1}$, and $\varphi_{f}\left(G_{f}\right) \subset G_{v}^{\prime}$ for $f \in \hat{E}(v) \backslash\{e\}$. Then, define $\mathcal{G}^{\prime}$ as follows:

- $V^{\prime}=V$;

- the oriented edge $\left\{e, e^{-1}\right\}$ is replaced with two oriented edges having the same endpoints as $e$ :

$$
\hat{E}^{\prime}=\hat{E} \cup\left\{e^{\prime}, e^{\prime \prime},\left(e^{\prime}\right)^{-1},\left(e^{\prime \prime}\right)^{-1}\right\} \backslash\left\{e, e^{-1}\right\}
$$

with $\partial_{0} e^{\prime}=\partial_{0} e^{\prime \prime}=\partial_{0} e$ and $\partial_{1} e^{\prime}=\partial_{1} e^{\prime \prime}=\partial_{1} e$;

- $G_{e^{\prime}}=G_{e}^{\prime}, G_{e^{\prime \prime}}=G_{e}^{\prime \prime}, G_{v}$ is redefined to be $G_{v}^{\prime}$; and

- $\varphi_{e^{\prime \prime}}=\left.i_{t^{-1}} \circ \varphi_{e}\right|_{G_{e}^{\prime \prime}}$ and other bonding maps are given by restricting domains and/or codomains of bonding maps of $\mathcal{G}^{\prime}$ if necessary.

We say that the new graph of groups is obtained from $\mathcal{G}$ by unkilling 5 See Figure 5

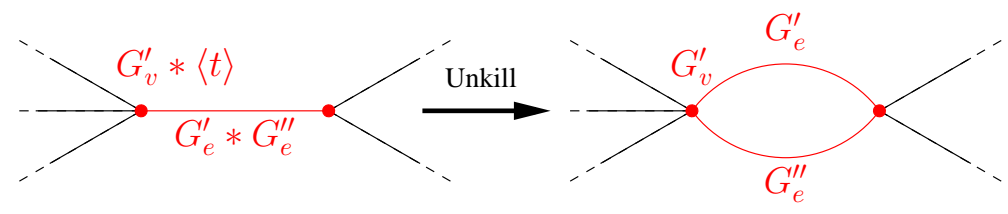

Figure 5: Unkilling

\footnotetext{
${ }^{5}$ We use the term unkilling because the inverse operation kills a cycle.
} 


\subsection{Cleaving}

Suppose that, for some $v \in V$ and $e \in \hat{E}(v), G_{v}=G_{v}^{\prime} * G_{v}^{\prime \prime}$ non-trivially, $G_{e}=G_{e}^{\prime} * G_{e}^{\prime \prime}, \varphi_{e}\left(G_{e}^{\prime}\right) \subset G_{v}^{\prime}, \varphi_{e}\left(G_{e}^{\prime \prime}\right) \subset G_{v}^{\prime \prime}$, and for $f \in \hat{E}(v) \backslash\{e\}$ either $\varphi_{f}\left(G_{f}\right) \subset G_{v}^{\prime}$ or $\varphi_{f}\left(G_{f}\right) \subset G_{v}^{\prime \prime}$. Then, define $\mathcal{G}^{\prime}$ as follows:

- $v$ is replaced by two vertices: $V^{\prime}=V \cup\left\{v^{\prime}, v^{\prime \prime}\right\} \backslash\{v\}$;

- the oriented edge $\left\{e, e^{-1}\right\}$ is replaced by two oriented edges:

$$
\hat{E}^{\prime}=\hat{E} \cup\left\{e^{\prime}, e^{\prime \prime},\left(e^{\prime}\right)^{-1},\left(e^{\prime \prime}\right)^{-1}\right\} \backslash\left\{e, e^{-1}\right\}
$$

with $\partial_{0} e^{\prime}=v^{\prime}, \partial_{0} e^{\prime \prime}=v^{\prime \prime}$, and $\partial_{1} e^{\prime}=\partial_{1} e^{\prime \prime}=\partial_{1} e$;

- for $f \in \hat{E}(v) \backslash\{e\}, \partial_{0} f=v^{\prime}$ if $\varphi_{e}\left(G_{f}\right) \subset G_{v}^{\prime}$ and $\partial_{0} f=v^{\prime \prime}$ if $\varphi_{e}\left(G_{f}\right) \subset$ $G_{v}^{\prime \prime}$

- $G_{v^{\prime}}=G_{v}^{\prime}, G_{v^{\prime \prime}}=G_{v}^{\prime \prime}, G_{e^{\prime}}=G_{e}^{\prime}, G_{e^{\prime \prime}}=G_{e}^{\prime \prime}$; and

- bonding maps are given by restricting domains and/or codomains of bonding maps of $\mathcal{G}^{\prime}$ if necessary.

We say that the new graph of groups is obtained from $\mathcal{G}$ by cleaving. See Figure 6. Each of the operations blowing up, unpulling, unkilling, and cleaving

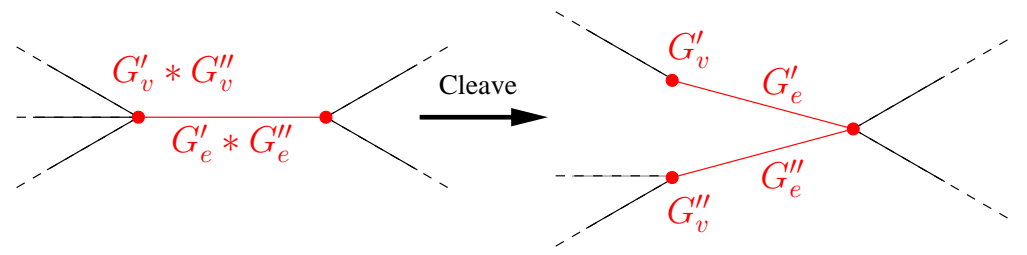

Figure 6: Cleaving

is a simplification.

Proposition 2.1 If $\mathcal{G}^{\prime}$ is obtained from $\mathcal{G}$ by reducing or simplifying, then $\pi_{1}\left(\mathcal{G}^{\prime}\right)$ and $\pi_{1}(\mathcal{G})$ are isomorphic.

Proof In the first type of reducing move, $\pi_{1}(\mathcal{G}) \cong \pi_{1}\left(\mathcal{G}^{\prime}\right) * G_{e} G_{e}$ where the map $G_{e} \rightarrow G_{e}$ is an isomorphism. By van Kampen's theorem, $\pi_{1}(\mathcal{G}) \cong \pi_{1}\left(\mathcal{G}^{\prime}\right)$. In all of the other cases, $\mathcal{G}$ is obtained from $\mathcal{G}^{\prime}$ by a Stallings fold and so $\pi_{1}(\mathcal{G}) \cong \pi_{1}\left(\mathcal{G}^{\prime}\right)$, see [4, Section 2].

Remark 2.2 If $\pi_{1}(\mathcal{G})$ is not infinite cyclic then the first type of blow up is a composition of a second type of blow up, an unkilling, and a reduction. Therefore, we will not have to consider the first type of blow up. 


\subsection{Our case}

Given a graph of groups, we want to iteratively simplify until the resulting graph of groups can't be simplified. In order to do this algorithmically, we need be able to recognize when a simplification is possible. To this end, we restrict the graphs of groups that we will consider to the case where $\Gamma$ is a finite graph, ie, where $E$ is finite, and where, for $v \in V$ and $e \in E, G_{v}$ and $G_{e}$ are finite rank free groups. Such a $\mathcal{G}$ is a finite graph of finite rank free groups.

\subsection{Labeled graphs}

Graphs will have two uses in this paper. The first we have already seen-these are as the underlying graphs of graphs of groups and are denoted by $\Gamma$ 's. The other use will be to represent subgroups of free groups and these will be denoted by $\Sigma$ 's. We now explain this second usage. Let $F_{\mathcal{B}}$ denote the free group with basis $\mathcal{B}$. For $S \subset F_{\mathcal{B}}, S^{ \pm 1}$ is defined to be $S \cup S^{-1}$ where $S^{-1}$ is $\left\{s^{-1}: s \in S\right\}$. A labeled graph or a $\mathcal{B}$-graph is a connected graph $\Sigma=\Sigma\left(V, \hat{E}\right.$, op, $\left.\partial_{0}\right)$ with a labeling function $\hat{E} \rightarrow \mathcal{B}^{ \pm 1}$ such that the label assigned to op $(c)=c^{-1}$ is the inverse of the label assigned to $c$. The $\mathcal{B}$-rose is a $\mathcal{B}$-graph $R_{\mathcal{B}}$ with one vertex and a bijective labeling function. We identify $\pi_{1}\left(R_{\mathcal{B}}\right)$ with $F_{\mathcal{B}}$. (The homotopy class of the path formed by the edge labeled $b$ is identified with $b$.) There is a natural map $\lambda_{\Sigma}: \Sigma \rightarrow R_{\mathcal{B}}$ sending 1-cells to 1-cells and preserving labels and orientations. Since $\lambda_{\Sigma}$ determines the labeling function and vice versa, we will call $\lambda_{\Sigma}$ the labeling function as well. The graph $\Sigma$ is based if there is a distinguished vertex $*$. On the level of fundamental groups, the image of $\lambda_{\Sigma}$ is a subgroup of $F_{\mathcal{B}}$ denoted $[(\Sigma, *)]$. If we forget the basepoint then the image is only defined up to conjugacy and $\Sigma$ determines a conjugacy class $[[\Sigma]]$ of subgroups $F_{\mathcal{B}}$. We say that $(\Sigma, *)$ represents $[(\Sigma, *)]$ and that $\Sigma$ represents $[[\Sigma]]$. More generally, if $\vec{\Sigma}$ is a sequence of labeled graphs then a sequence $[[\vec{\Sigma}]]$ of conjugacy classes of subgroups of $F_{\mathcal{B}}$ is determined. If there are basepoints $\vec{*}$ then a sequence $[(\vec{\Sigma}, \vec{*})]$ of subgroups of $F_{\mathcal{B}}$ is determined.

It is well-known, see eg [16, Section $1 . \mathrm{A}]$, that a generating set $[\Sigma, *]$ may be obtained as follows. Choose a maximal tree $T$ for $\Sigma$ and choose orientations for the edges not in $T$. The generating set is indexed by these oriented edges. Specifically, the generator corresponding to the oriented edge $c$ is the word in $\mathcal{B}^{ \pm 1}$ determined by reading the labels of the loop obtained by concatenating the path in $T$ from $*$ to $\partial_{0} c, c$, and the path in $T$ from $\partial_{1} c$ back to $*$. 


\subsection{Stallings and Gersten representatives}

The complexity $\mathrm{c}(\Sigma)$ of the labeled graph $\Sigma$ is $|E(\Sigma)|$ and the complexity $\mathrm{c}(\vec{\Sigma})$ of the sequence $\vec{\Sigma}=\left\{\Sigma_{i}\right\}_{i \in I}$ of labeled graphs is $\sum_{i \in I} \mathrm{c}\left(\Sigma_{i}\right)$. If $\vec{H}$ is a finite sequence of finitely generated subgroups of $F_{\mathcal{B}}$, the Stallings representative for $\vec{H}$ with respect to $\mathcal{B}$ is the sequence $\vec{\Sigma}_{\mathrm{S}}=\Sigma_{\mathrm{S}}(\vec{H}, \mathcal{B})$ of based $\mathcal{B}$-graphs of minimal complexity representing $\vec{H}$. We often omit the $\mathcal{B}$ from the notation. If $\vec{H}$ is represented by a finite sequence $\vec{H}=\left\{H_{i}=\left\langle S_{i}\right\rangle\right\}$ where each $S_{i}$ is a finite set of words in $\mathcal{B}^{ \pm 1}$, then there is an algorithm due to Stallings [27] to find $\vec{\Sigma}_{\mathrm{S}}$ from $\left\{S_{i}\right\}$, see also 10 .

If $\overrightarrow{\mathcal{H}}$ is a sequence of conjugacy classes of non-trivial subgroups of $F_{\mathcal{B}}$, the Stallings representative for $\overrightarrow{\mathcal{H}}$ with respect to $\mathcal{B}$ is the $\mathcal{B}$-graph $\Sigma_{\mathrm{S}}(\overrightarrow{\mathcal{H}})$ of minimal complexity representing $\overrightarrow{\mathcal{H}}$. In fact, if $\overrightarrow{\mathcal{H}}$ is represented by $\vec{H}=\left\{H_{i}=\left\langle S_{i}\right\rangle\right\}$ as above then $\Sigma_{\mathbf{S}}(\overrightarrow{\mathcal{H}})$ is the sequence core $\left(\Sigma_{\mathbf{S}}(\vec{H})\right)$ of cores of elements of $\Sigma_{\mathbf{S}}(\vec{H})$. Recall that if $\Sigma$ is graph then the core of $\Sigma$, denoted core $(\Sigma)$, is the union of all immersed circuits in $\Sigma$, see also Section 9.2.1 In particular, there is an algorithm to find core $\left(\Sigma_{\mathrm{S}}(\vec{H})\right)$ from $\left\{S_{i}\right\}$ as well as a sequence $\vec{h}$ of elements of $F_{\mathcal{B}}$ such that $\operatorname{core}\left(\Sigma_{\mathrm{S}}(\vec{H})\right)=\Sigma_{\mathrm{S}}\left(\vec{H}^{\vec{h}}\right)$ where $\vec{H}^{\vec{h}}$ is the sequence of groups obtained by conjugating a component of $\vec{H}$ with the corresponding component of $\vec{h}$. In fact, if $\Sigma$ is a component of $\Sigma_{\mathrm{S}}(\vec{H})$ then the corresponding component $h$ of $\vec{h}$ can be taken to be the inverse of the word read along the shortest path from the basepoint $*$ of $\Sigma$ to core $(\Sigma)$. It is convenient to also allow conjugacy classes of trivial groups in $\overrightarrow{\mathcal{H}}$. Since the core of a tree is empty, we take the Stallings representative of the conjugacy class of the trivial group to be the empty set.

A Gersten representative $\Sigma_{\mathrm{G}}(\overrightarrow{\mathcal{H}})$ for $\overrightarrow{\mathcal{H}}$ is a sequence of $\mathcal{B}$-graphs of minimal complexity among sequences of $\mathcal{B}$-graphs representing $\alpha \overrightarrow{\mathcal{H}}$ as $\alpha$ varies over $\operatorname{Aut}\left(F_{\mathcal{B}}\right)$. (If $\vec{H}$ represents $\overrightarrow{\mathcal{H}}$, then $\alpha \vec{H}$ represents $\alpha \overrightarrow{\mathcal{H}}$, where $\alpha$ is applied coordinate-wise.) If $\overrightarrow{\mathcal{H}}$ is represented by $\vec{H}=\left\{H_{i}=\left\langle S_{i}\right\rangle\right\}$ as above, then there is an algorithm that produces a $\Sigma_{\mathrm{G}}(\overrightarrow{\mathcal{H}})$ as well as an automorphism $\alpha$ such that $\operatorname{core}\left(\Sigma_{\mathrm{S}}(\alpha \vec{H})\right)=\Sigma_{\mathrm{G}}(\overrightarrow{\mathcal{H}})$, see [14, 18, and also Section 9.5]

Example 2.3 If $\mathcal{B}=\{a, b\}$ and if $H=\left\langle a a b a^{-1}, a b^{-1} a b b a^{-1}\right\rangle$, then the graph $(\Sigma, *)$ pictured in Figure 7 represents $H$. (The open arrows denote ' $a$ ' and the closed ' $b$ '.) The graph $\left(\Sigma_{\mathrm{S}}, *\right)$ is the Stallings representative of $H$. For the automorphism $\alpha: F_{\mathcal{B}} \rightarrow F_{\mathcal{B}}$ given by $a \mapsto a b^{-1}, b \mapsto b$, a Gersten representative $\Sigma_{\mathrm{G}}$ of $H$ is the core of the Stallings representative for $\alpha H$. 

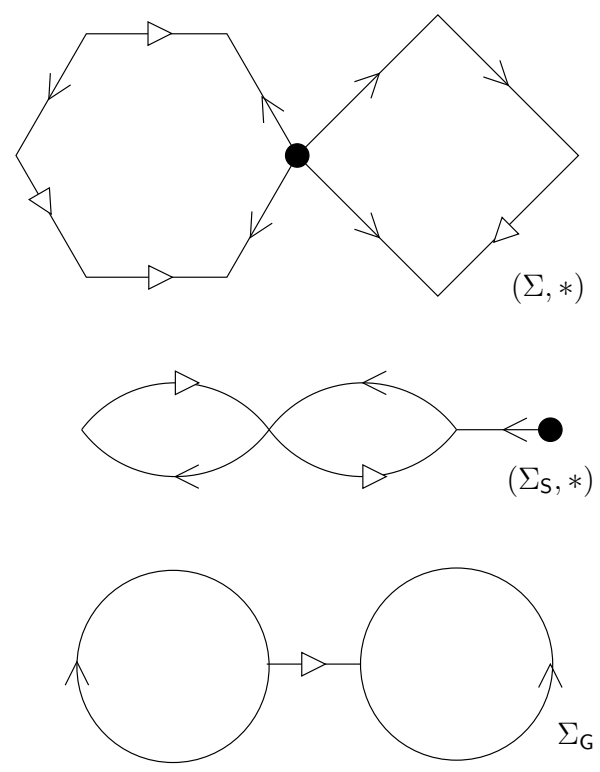

Figure 7

Notation 2.4 If $\vec{\Sigma}^{\prime}=\left\{\Sigma_{i}^{\prime}\right\}_{i \in I}$ and $\vec{\Sigma}^{\prime \prime}=\left\{\Sigma_{j}^{\prime \prime}\right\}_{j \in J}$ are sequences of $\mathcal{B}$-graphs and if $\{0\}=I \cap J$ then $\vec{\Sigma}^{\prime} \vee \vec{\Sigma}^{\prime \prime}=\left\{\Sigma_{k}\right\}_{k \in I \cup J}$ is a sequence of graphs with labels in $\mathcal{B}$ of the following form:

$$
\Sigma_{k}= \begin{cases}\Sigma_{0}^{\prime} \vee \Sigma_{0}^{\prime \prime}, & \text { if } k=0 ; \\ \Sigma_{i}^{\prime}, & \text { if } k \in I \backslash\{0\} ; \text { and } \\ \Sigma_{j}^{\prime \prime}, & \text { if } k \in J \backslash\{0\} .\end{cases}
$$

Definition 2.5 Let $\vec{\Sigma}=\left\{\Sigma_{i}\right\}_{i \in I}$ be a sequence of $\mathcal{B}$-graphs.

(1) If there is a non-trivial partition $\mathcal{B}=\mathcal{B}^{\prime} \sqcup \mathcal{B}^{\prime \prime}$ such that, for each $i \in I$, the labels of $\Sigma_{i}$ are either all in $\mathcal{B}^{\prime}$ or all in $\mathcal{B}^{\prime \prime}$, then we say that $\vec{\Sigma}$ can be visibly blown up.

(2) Suppose that $b \in \mathcal{B}$ appears as a label in only one element $\Sigma$ of $\vec{\Sigma}$ and that only one oriented edge $c_{0}$ of $\Sigma$ has label $b$. Suppose further that $c_{0} \subset \operatorname{core}(\Sigma)$.

(a) If $c_{0}$ does not separate $\Sigma$ then we say that $\vec{\Sigma}$ can be visibly unpulled.

(b) If $c_{0}$ does separate $\Sigma$ then we say that $\Sigma$ can be visibly unkilled.

(3) If there is a non-trivial partition $\mathcal{B}=\mathcal{B}^{\prime} \sqcup \mathcal{B}^{\prime \prime}$ such that $\vec{\Sigma}=\vec{\Sigma}^{\prime} \vee \vec{\Sigma}^{\prime \prime}$ where $\vec{\Sigma}^{\prime}$ is a sequence of $\mathcal{B}^{\prime}$-graphs and $\vec{\Sigma}^{\prime \prime}$ is a sequence of $\mathcal{B}^{\prime \prime}$-graphs then we say that $\vec{\Sigma}$ can be visibly cleaved. 
If $\vec{\Sigma}$ can be visibly blown up, visibly unpulled, visibly unkilled, or visibly cleaved, then we say that $\vec{\Sigma}$ can be visibly simplified.

After the statement of the next proposition, we can describe the algorithm. The proof of this proposition is almost obvious, but requires some bookkeeping which is postponed until the appendix. This proposition is subsumed into Proposition A.3.

Notation 2.6 Let $\mathcal{G}$ be a graph of finite rank free groups with notation as in Section 2.1] For $v \in V$, let $\vec{H}(v)$ denote the sequence of subgroups of $G_{v}$ represented by $\left\{\varphi_{e}\left(G_{e}\right): e \in \hat{E}(v)\right\}$. Also let $\overrightarrow{\mathcal{H}}(v)$ denote the sequence of conjugacy classes of subgroups of $G_{v}$ represented by $\vec{H}(v)$.

Proposition 2.7 Suppose that for some $v \in V, \Sigma_{\mathrm{G}}(\overrightarrow{\mathcal{H}}(v))$ can be visibly simplified. Then, $\mathcal{G}^{\text {out }} \sim \mathcal{G}$ can be algorithmically found such that $\mathcal{G}^{\text {out }}$ can be simplified.

\subsection{The algorithm}

Here is the algorithm. See Figure 8 for a flow chart. More details on the algorithm are given in Section 9] Section [10] and the appendix.

Step 0 Input $\mathcal{G}$, a finite graph of finite rank free groups.

Step 1 Reduce $\mathcal{G}$.

Step 2 If, for some $v \in V, \Sigma_{\mathrm{G}}(\overrightarrow{\mathcal{H}}(v))$ can be visibly simplified, then replace $\mathcal{G}$ by a simplified conjugate and return to Step 1 . Else, done.

The main result of the paper is:

Theorem 2.8 Suppose that a finite graph $\mathcal{G}$ of finite rank free groups is input into the above algorithm and that $\mathcal{G}^{\text {out }}$ is output. Then, the decomposition of $\pi_{1}(\mathcal{G})$ determined by the edges of $\mathcal{G}^{\text {out }}$ with trivial stabilizer is the Grushko decomposition of $\pi_{1}(\mathcal{G})$.

The proof of Theorem 2.8 is found in Section 10. 


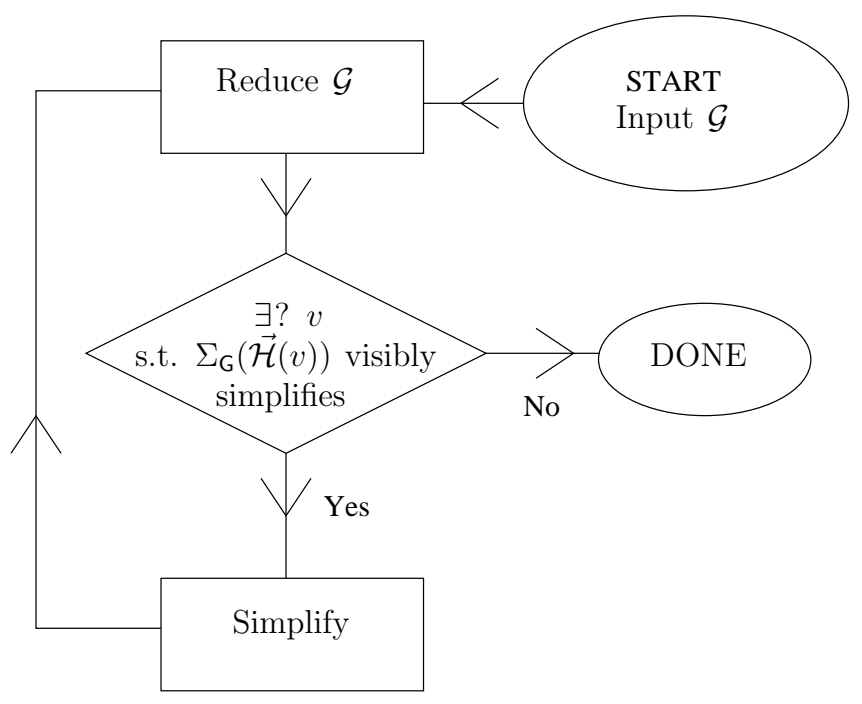

Figure 8: Flow chart

\subsection{Relative version}

In this section we describe a relative version of Theorem 2.8 If $H$ is a subgroup of a group $G$, then we say that $G$ is freely decomposable rel $H$ if there is a free decomposition $G=G^{\prime} * G^{\prime \prime}$ with $H \subset G^{\prime}$ and $G^{\prime \prime}$ non-trivial. Otherwise, $G$ is freely indecomposable rel $H$. The relative version of Grushko's theorem (Theorem 1.1) is:

Theorem 2.9 Suppose $H$ is a subgroup of the finitely generated group $G$. Then, $G$ is a free product $G_{H} * G_{1} * \cdots * G_{n} * F$ where $H \subset G_{H}, G_{H}$ is freely indecomposable rel $H, G_{i}$ for $1 \leq i \leq n$ is freely indecomposable and not free, and $F$ is a finite rank free group.

The subgroup $G_{H}$ is unique in this the Grushko decomposition of $G$ rel $H$. Up to reordering and conjugation, the $G_{i}, 1 \leq i \leq n$, are unique. Also, the rank of $F$ is an invariant of the pair $H \subset G$.

Suppose now that $\mathcal{G}$ is a finite graph of finite rank free groups, that $v_{0} \in V$ has valence one with incident edge $e_{0}$, and that $\varphi_{e_{0}}$ is an isomorphism. We are going to describe a slight modification of the algorithm of Section 2.10] that produces the Grushko decomposition of $\pi_{1}(\mathcal{G})$ rel $G_{v_{0}}$. Intuitively, in the modified algorithm we only reduce or visibly simplify only if the special edge group $G_{e_{0}}$ is unchanged. Specifically, we modify the algorithm as follows. 
Steps 0 and 1 are replaced by:

Step $0^{\prime}$ Input $\mathcal{G}$, a finite graph of finite rank free groups as above.

Step $1^{\prime}$ Reduce $\mathcal{G}$ rel $v_{0}$, ie, apply the reducing moves displayed in Figure 1 only if $e \notin\left\{e_{0}, e_{0}^{-1}\right\}$.

To describe the modification of Step 2, we need a definition. In Definition 2.5)(2), the component $\Sigma$ of $\vec{\Sigma}$ is special. In Definition 2.5)(3), the component of $\vec{\Sigma}$ corresponding to $\Sigma_{0}=\Sigma_{0}^{\prime} \vee \Sigma_{0}^{\prime \prime}$ in Notation 2.4 is special. In Definition 2.5)(11), none of the components of $\vec{\Sigma}$ are special. The components of $\Sigma_{\mathrm{G}}(\overrightarrow{\mathcal{H}}(v))$ are parametrized by the set $\hat{E}(v)$ of edges incident to $v$. If $\Sigma_{\mathrm{G}}(\overrightarrow{\mathcal{H}}(v))$ can be visibly simplified, then the edge corresponding to the special component is special. If the special edge $e$ is not in $\left\{e_{0}, e_{0}^{-1}\right\}$, then the resulting simplification will not change $G_{e_{0}}$. Step 2 of the algorithm is replaced by:

Step $2^{\prime}$ If, for some $v \in V, \Sigma_{\mathrm{G}}(\overrightarrow{\mathcal{H}}(v))$ can be visibly simplified and the special edge is not in $\left\{e_{0}, e_{0}^{-1}\right\}$, then replace $\mathcal{G}$ by a simplified conjugate and return to Step $1^{\prime}$. Else, done.

Theorem 2.10 Suppose that $\mathcal{G}$ as above is input into the modified algorithm and that $\mathcal{G}^{\text {out }}$ is output. Then, the decomposition of $\pi_{1}(\mathcal{G})$ determined by the edges of $\mathcal{G}^{\text {out }}$ with trivial stabilizer gives the Grushko decomposition of $\pi_{1}(\mathcal{G})$ rel $G_{v_{0}}$.

The proof of the relative version requires only minor notational changes to the proof of Theorem 2.8 and is left to the reader.

\section{Laminated square complexes and models}

This section contains a discussion of certain laminated two complexes called models whose $2-$ cells are squares. For an interesting study of complexes built from squares see [6].

Let $I$ denote the unit interval $[0,1]$. An $n$-cube is a metric space isometric to $I^{n}$. A metric space is a cube if it is an $n$-cube for some $n$. A cube complex is a union of cubes glued by isometries of faces. A finite dimensional cube complex $X$ admits a maximal metric such that the inclusion $C \rightarrow X$ is a local isometry for each cube $C$ of $X$ [5]. A square is a 2-cube, and we only have need to consider square complexes, ie, cube complexes of dimension at most two. A 
graph is a 1-dimensional cube complex 6 , a tree is a simply connected graph and a forest is a disjoint union of trees.

A decomposition of a square is standard if it is induced by projection to a codimension-1 face. A decomposition of a 1-cube is standard if all decomposition elements are points. It is trivial if the only decomposition element is the 1-cube itself. A laminated square complex is a simply connected square complex $X$ with a decomposition $\mathcal{D}$ such that:

(M1) The link of every vertex of $X$ is a flag complex.

(M2) For each square $C$ of $X$, the induced decomposition of $C$ is standard. In other words, the decomposition of $C$ whose elements are the components of $C$ intersected with elements of $\mathcal{D}$ is standard. For each 1 -cube of $X$, the induced decomposition is either standard or trivial.

In this context, (M1) means that every link is a simplicial graph with no circuits of length three. A decomposition element is also called a leaf.

Proposition 3.1 Let $(X, \mathcal{D})$ be a laminated square complex. Then,

(1) $X$ is contractible and

(2) leaves are forests.

Proof (M1) implies that the the metric on $X$ is $C A T(0)$ [5]. Hence, (1). A vertex of a square with a standard decomposition is contained in exactly one edge that is contained in a leaf. So, links of vertices in $X$ are bipartite and leaves are totally geodesic. In particular, leaves are 1-dimensional and contractible.

A model is a laminated square complex $(X, \mathcal{D})$ such that:

(M3) Leaves are connected.

The next proposition is immediate from definitions.

Proposition 3.2 Let $(X, \mathcal{D})$ be a laminated square complex and let $\hat{\mathcal{D}}$ be the decomposition of $X$ whose elements are the connected components of elements of $\mathcal{D}$. Then, $(X, \hat{\mathcal{D}})$ is a model.

\footnotetext{
${ }^{6} 1$-dimensional $C W$-complexes and 1-dimensional cube complexes are both called graphs. Since we will only be using combinatorial properties, the distinction is not important to us.
} 
Definition 3.3 We say that $(X, \hat{\mathcal{D}})$ is induced by $(X, \mathcal{D})$.

Proposition 3.4 If $(X, \mathcal{D})$ is a model, then the decomposition space $X / \mathcal{D}$ is a tree.

Proof Since a leaf is totally geodesic, it intersects each square $C$ in a connected set. In particular, the decomposition $C \cap \mathcal{D}$ of $C$ obtained by intersecting $C$ with elements of $\mathcal{D}$ is standard, $I=C / C \cap \mathcal{D} \rightarrow X / \mathcal{D}$ is injective, and $X / \mathcal{D}$ is naturally a graph. Leaves are connected, and so $X \rightarrow X / \mathcal{D}$ is $\pi_{1}$-surjective. Thus, $X / \mathcal{D}$ is a tree.

If $(X, \mathcal{D})$ is a model, we say that $X$ is a model for the tree $S=X / \mathcal{D}$ or that $X \rightarrow S$ is a model. The preimage in $X$ of $s \in S$ is $X_{s}$. If $c$ is an edge of $S$ and if $s, s^{\prime} \in \stackrel{\circ}{c}$, then $X_{s}$ and $X_{s^{\prime}}$ have the same isomorphism type $X_{c}$. We will sometimes abuse notation and identify $X_{c}$ with $X_{s}$ for $s \in \stackrel{\circ}{c}$.

Notation 3.5 If the group $G$ acts on the set $X$ and if $S$ is a subset of $X$, then $G_{X, S}$ is the stabilizer of $S$, ie, the subgroup elements $g \in G$ such that $g(S)=S$. If $S=\{s\}$ then we also write $G_{X, s}$ for $G_{X, S}$. We will suppress the $X$ if the space is understood.

An action of a group $G$ on $(X, \mathcal{D})$ is an (isometric) action of $G$ on $X$ permuting cubes and decomposition elements. In this case, $(X, \mathcal{D})$ is a $G$-model. There is an induced action of $G$ on $S=X / \mathcal{D}$. The quotients $X / G$ and $S / G$ are denoted $\bar{X}$ and $\bar{S}$ respectively. We say that $G$ acts without inversions if, for all cubes $C \subset X, G_{C}$ fixes $C$ pointwise. By subdividing $X$, we may arrange that $G$ acts without inversions. Hence, we always assume that our actions are without inversions. Note that the space $X_{s}$ is $G_{s}$-invariant and $X_{c}$ is $G_{c}$-invariant.

\section{Trees}

We review some tree basics. A $G$-tree $S$ is minimal if it has no proper $G-$ invariant subtrees. It is trivial if has a fixed point. In this case we also say that the $G$-action is elliptic. If $S$ has a unique minimal invariant $G$-subtree, then it is denoted $S_{G}$. This occurs, for example, if $G$ contains a hyperbolic element, that is an element that fixes no point of $S$ [1. A morphism $S \rightarrow T$ of $G$-trees is a simplicial $G$-map. It is strict if no edge is mapped to a point. If there is a morphism $S \rightarrow T$, then $S$ resolves $T$. 
If $e$ is an edge of $S$, then we say that $S^{\prime}$ is obtained from $S$ by collapsing $e$ if $S^{\prime}$ is the result of equivariantly collapsing $e$. A morphism $S \rightarrow S^{\prime}$ is a collapse if $S^{\prime}$ is obtained from $S$ by iteratively collapsing edges. If edges $e_{1}$ and $e_{2}$ of $S$ share the vertex $s$, then we say that $S^{\prime}$ is obtained from folding $e_{1}$ and $e_{2}$ if $S^{\prime}$ is the result of equivariantly identifying $e_{1}$ and $e_{2}$ with an isometry fixing $s$. The resulting morphism $S \rightarrow S^{\prime}$ is a fold.

\section{$5 \quad$ Examples of Models}

Example 5.1 First a non-example. Glue two squares along three sides and laminate so that restrictions to squares are standard and so that the unglued sides form a leaf. The result is not a laminated square complex even though it is contractible (there are vertices whose links consist of distinct edges with the same endpoints-such a link is not a simplicial graph). Notice that not all leaves are trees.

Example 5.2 The quotient $\bar{X} \rightarrow \bar{S}$ of a $G$-model $X \rightarrow S$ with $G$ free of rank 2 is depicted in Figure 9 . The preimage in $\bar{X}$ of a point in the interior of the edge of $\bar{S}$ is isomorphic to a circle. The preimage of the vertex is a "pair of eyeglasses'. The stabilizer of a vertex of $S$ is free of rank two; the stabilizer of an edge of $S$ is infinite cyclic.

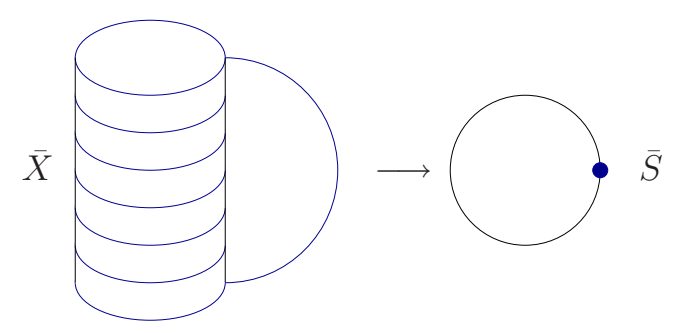

Figure 9

Example 5.3 If $S$ and $T$ are $G$-trees, then

$$
S \times T=\cup\{e \times f: e \text { is an edge of } S, f \text { is an edge of } T\}
$$

is a union of squares with projection maps $q_{S}: S \times T \rightarrow S$ and $q_{T}: S \times T \rightarrow T$. The induced decomposition with quotient $S$ (respectively $T$ ) gives $S \times T$ the structure of a model for $S$ (respectively $T$ ). 
Example 5.4 Let $(X, \mathcal{D})$ be a $G$-model and let $Y$ be a simply connected $G$ subcomplex of $X$. Then, $Y$ with the decomposition $\mathcal{D}(Y)=\{D \cap Y \mid D \in \mathcal{D}\}$ is a laminated square complex and $Y / \mathcal{D}(Y) \rightarrow X / \mathcal{D}$ is an inclusion. If we let $\hat{\mathcal{D}}(Y)$ be the decomposition of $Y$ induced by $\mathcal{D}(Y)$ (see Definition [3.3), then $(Y, \hat{\mathcal{D}}(Y)))$ is a model and $Y / \hat{\mathcal{D}}(Y) \rightarrow X / \mathcal{D}$ is a morphism. We call $\hat{\mathcal{D}}(Y)$ the restricted decomposition of $Y$.

Example 5.5 Main Example Suppose that $S$ and $T$ are $G$-trees and that, for each $s \in S$, there is a unique minimal $G_{s}$-invariant subtree $T_{s}$ of $T$. The union $X_{S}(T)=\bigsqcup_{s \in S}\left(\{s\} \times T_{s}\right)$ is a subcomplex of $S \times T$ and is simplyconnected (being a union of simply-connected spaces along simply-connected spaces). $\quad X_{S}(T) \rightarrow S$ is a model as is $X_{S}(T) \rightarrow \hat{T}$ where $\hat{T}$ is the quotient of the decomposition induced from $X_{S}(T) \subset S \times T \rightarrow T$ by restriction (see Example 5.4). If $\bar{S}=S / G$ and all $\bar{T}_{s}=T_{s} / G_{s}$ are compact, then $\overline{X_{S}(T)}=$ $X_{S}(T) / G$ is also compact.

Proposition 5.6 $\hat{T}$ as in Example 5.5 is minimal.

Proof We may identify $T_{s}$ with the image of the injection $\{s\} \times T_{s} \rightarrow \hat{T}$. By construction, $\hat{T}=\cup_{s \in S} T_{s}$. If $\hat{t} \in \hat{T}$ is not contained in an invariant $G$-subtree $R$ of $\hat{T}$ and if $\hat{t} \in T_{s}$, then $R \cap T_{s}$ is a proper $G_{s}$-invariant subtree of $T_{s}$, contradiction.

\section{Operations on models}

In this section, we assume that $G$ is a group, $S$ is a $G$-tree, and $X \rightarrow S$ is a model. We will describe operations on $X$. In each case, the result $X^{\prime}$ is a model for $S^{\prime}$ where $S^{\prime}$ resolves $S$. The operations are geometric generalizations of the simplifications of Section 2

\section{$6.1 \quad 0-$ Simplifying}

Let $C=I \subset X$ be a $1-$ cube and set $C_{0}=\{0\}$. Suppose that

- $C$ meets cubes of $X$ other than faces of $C$ only in $\{1\}$; and

- the restriction of the decomposition to $C$ is $\{C\}$.

Let $X^{\prime}$ be the result of equivariantly replacing $C$ by $\{1\}$, ie,

$$
X^{\prime}=X \backslash \cup_{g \in G} g \cdot[0,1) .
$$

We say that $X^{\prime}$ with the restricted decomposition is obtained from $X$ by $0_{-}$ simplifying $C$ from $C_{0}$. Here $S^{\prime}=S$. See Figure 10. 


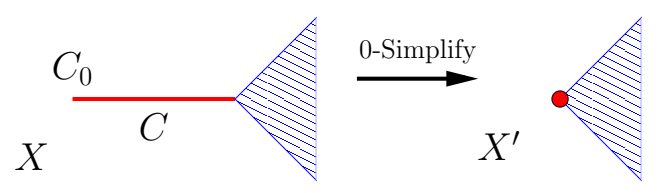

Figure 10

\section{$6.2 \quad$ I-Simplifying}

Let $C=I \times I^{n} \subset X(n=0$ or 1$)$ be a cube and set $C_{0}=\{0\} \times I^{n}$. Suppose that

- $C$ meets cubes of $X$ other than faces of $C$ only in $\{1\} \times I^{n}$; and

- $C_{0}$ is a decomposition element.

Let $X^{\prime}$ be the result of equivariantly replacing $C$ by $\{1\} \times I^{n}$. We say that $X^{\prime}$ with the restricted decomposition is obtained from $X$ by $I$-simplifying $C$ from $C_{0} . S \rightarrow S^{\prime}$ is a collapse. See Figure 11.

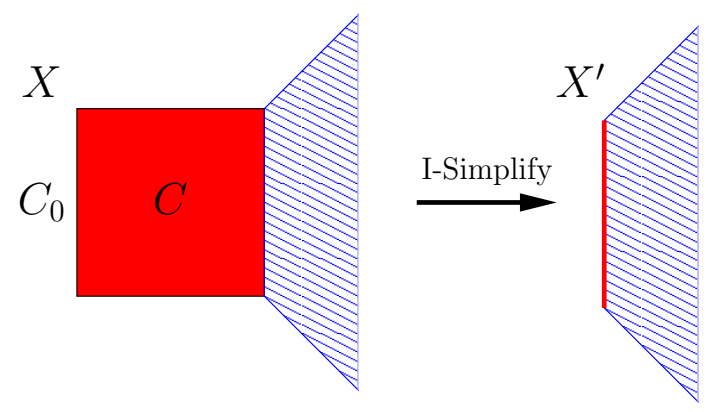

Figure 11

\subsection{II-Simplifying}

Let $C=I^{2} \subset X$ be a square and set $C_{0}=\{0\} \times I$. Suppose that

- $C$ meets cubes of $X$ other than faces of $C$ only in

$$
(\{1\} \times I) \cup(I \times\{1\}) ;
$$

- it is not possible to I-simplify from $C_{0}$, ie, $[0,1) \times\{1\}$ meets a cube other than a face of $C$; and

- $C_{0}$ is an element of the decomposition restricted to $C$. 
The model $X^{\prime}$ with restricted decomposition elements obtained by equivariantly replacing $C$ by

$$
(\{1\} \times I) \cup(I \times\{1\})
$$

is the result of $I I$-simplifying $C$ from $C_{0}$. Note that $S^{\prime}=S$. See Figure 12

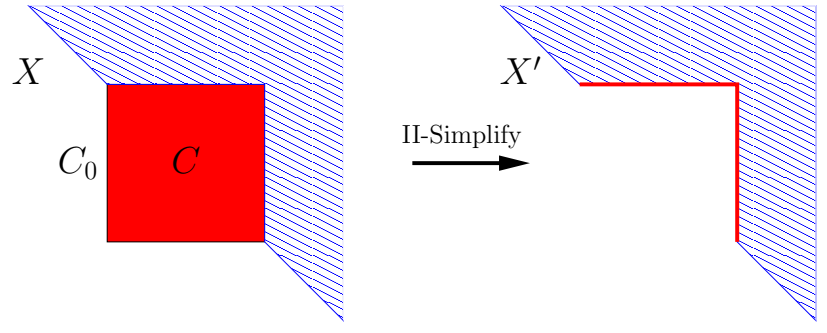

Figure 12

\subsection{III-Simplifying}

Let $C=I^{2} \subset X$ be a square and set $C_{0}=\{0\} \times I$. Suppose that

- $C$ meets cubes of $X$ other than faces of $C$ only in $(\partial I \times I) \cup(I \times\{1\})$;

- it is not possible to I-, or II-simplify from $C_{0}$; and

- $C_{0}$ is an element of the decomposition restricted to $C$.

The model $X^{\prime}$ with restricted decomposition elements obtained by equivariantly replacing $C$ by $(\partial I \times I) \cup(I \times\{1\})$ is the result of III-simplifying $C$ from $C_{0}$. Note that $S^{\prime} \rightarrow S$ is a non-trivial fold. See Figure 13
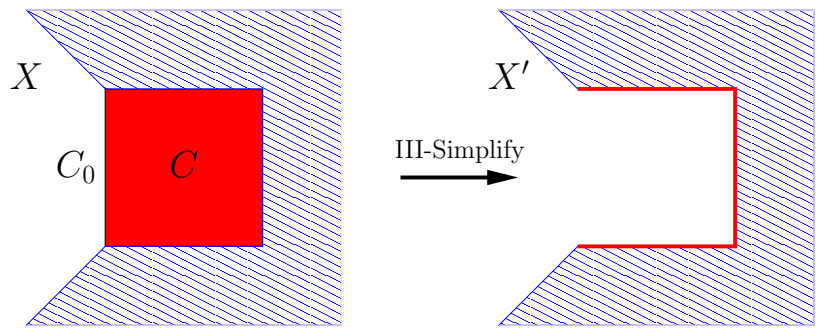

Figure 13 


\subsection{Blowing up}

If there is a cube $C=I \subset X$ such that $\stackrel{\circ}{I}$ meets no cube other than faces of $C$ and such that the decomposition restricted to $C$ is $\{C\}$ then we may refine the decomposition by $G$-equivariantly replacing the decomposition element $X_{C}$ containing $C$ with

$$
\left\{\{g \cdot t\} \mid t \in \stackrel{\circ}{I}, g \in G_{X_{C}}\right\} \sqcup\left\{\text { components of } X_{C} \backslash G_{X_{C}} \cdot \stackrel{\circ}{I}\right\} .
$$

We say that $X^{\prime} \rightarrow S^{\prime}$ is obtained from $X \rightarrow S$ by blowing up $C$. The induced map $S^{\prime} \rightarrow S$ collapses to points the edges of $S^{\prime}$ corresponding to the orbit of $C$, explaining the term "blowing up". See Figure 14.

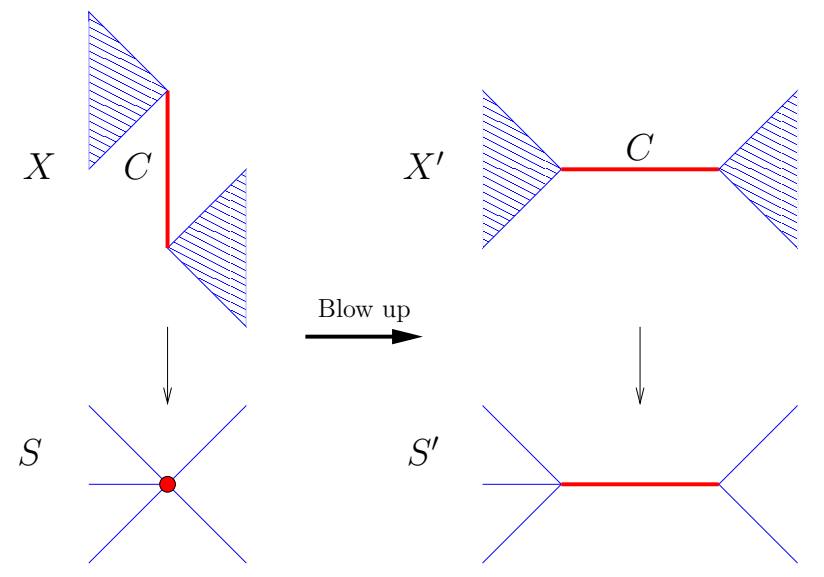

Figure 14

Definition 6.1 Let $S^{\prime} \rightarrow S$ a morphism. If there is a model $Y \rightarrow S$ and a cube $C$ of $Y$ with face $C_{0}$ such that 0 -simplifying $C$ from $C_{0}$ yields $Y^{\prime} \rightarrow S^{\prime}$, then we say that $S^{\prime}$ is obtained from $S$ by $0-G_{0}$-simplifying or equivalently by 0-simplifying over $G_{0}$ where $G_{0}$ is the stabilizer of $C_{0}$ in $Y$. The definitions for I- $G_{0^{-}}$, II- $G_{0^{-}}$, and III- $G_{0}$-simplifying are analogous. If there is a model $Y \rightarrow S$ with a cube $C$ such that $Y^{\prime} \rightarrow S^{\prime}$ is the result of blowing up $C$, then we say that $S^{\prime}$ is obtained from $S$ by $G_{0}$-blowing up or equivalently by blowing up over $G_{0}$ where $G_{0}$ is the stabilizer of $C$ in $Y$. In each of these cases, we say that $S^{\prime}$ is obtained from $S$ by simplifying over $G_{0}$ or just by simplifying if $G_{0}$ is understood.

Remark 6.2 Since the identity map $S \rightarrow S$ is an example of a model, blowing up as defined in Section 2.3 is an example of 1 -blowing up. 
The Grushko decomposition of a finite graph of free groups

1857

\section{Generalized Shenitzer-Swarup}

In the next lemma, we use the notation of Example 5.5.

Lemma 7.1 Suppose that $G$ is a group and that $S$ and $T$ are $G$-trees such that

(1) $\bar{S}$ is compact;

(2) for each $s \in S$ there is a unique minimal cocompact $G_{s}$-subtree $T_{s}$ of $T$; and

(3) for each edge $f \subset T$, the action of $G_{f}$ on $S$ is elliptic.

Then, there is a sequence

$$
\left\{X_{s}(T)=X_{0} \rightarrow S=S_{0}, X_{1} \rightarrow S_{1}, \cdots, X_{N} \rightarrow S_{N}\right\}
$$

of $I-, I I-$, and III-simplifications such that $\left(X_{N}\right)_{\hat{f}}$ is a point for each edge $\hat{f}$ of $\hat{T}$. Further, all the simplifications are over subgroups of edge stabilizers of $T$.

Proof Recall from Example 5.5 that $X_{0} \rightarrow \hat{T}$ is obtained by restricting $S \times$ $T \rightarrow T$. It is not possible to 0 -simplify $X_{0} \rightarrow S_{0}$. Indeed, in order to 0 -simplify $X_{0}$ there would have to be a 1 -cube as in the definition of 0 -simplifying. By the construction of $X_{0}$, the restriction of the decomposition giving $X_{0} \rightarrow \hat{T}$ to this $1-$ cube is standard. This is impossible since, by Proposition [5.6] $\hat{T}$ is minimal. Further, if $X_{i}$ is obtained from $X_{0}$ by a sequence of I-, II-, and IIIsimplifications, then the restriction to $X_{i}$ of the decomposition giving $X_{0} \rightarrow$ $\hat{T}$ still has decomposition space $\hat{T}$. (It is the decomposition space $S_{i}$ of the restriction of $S \times T \rightarrow S$ that can change.) In particular, it is also not possible to 0-simplify $X_{i}$.

Suppose we have constructed the sequence

$$
\left\{X_{S}(T)=X_{0} \rightarrow S=S_{0}, X_{1} \rightarrow S_{1}, \cdots, X_{i} \rightarrow S_{i}\right\} .
$$

We will describe how to proceed. Let $\hat{t}$ be a point in the interior of an edge of $\hat{T}$. The preimage $\left(X_{i}\right)_{\hat{t}}$ of $\hat{t}$ under $X_{i} \rightarrow \hat{T}$ is a $G_{\hat{t}}$-subtree of $S \times\{\hat{t}\}$. By (1) and (2), $X_{0}$, and so also $X_{i}$, is cocompact. Therefore, $\left(X_{i}\right)_{\hat{t}} / G_{\hat{t}}$ is compact. Since $T^{\prime}$ resolves $T$, by (3) the action of $G_{\hat{t}}$ on $S \times\{\hat{t}\}$, and hence also on $X_{\hat{t}}$ is elliptic. We see that $X_{\hat{t}} / G_{\hat{t}}$ is a finite tree. If this finite tree is not a single vertex then $X_{\hat{t}}$ contains a valence one vertex whose stabilizer equals the stabilizer of the incident edge. 
If there is such a valence one vertex, then this vertex is contained in a cube $C_{0}^{i}=I \subset X_{i}$ that projects to a point in $S_{i}$. In this case, simplify from $C_{0}^{i}$ to obtain $X_{i+1} \rightarrow S_{i+1}$. Stop if, for each edge $\hat{f}$ of $\hat{T}, \hat{T}_{\hat{f}}$ is a point.

The process must eventually stop since there are only finitely many $G$-orbits of cubes in $X_{S}(T)$.

The final claim of the lemma follows from the observation that $C_{0}^{i}$ projects to an edge of $\hat{T}$ and so the stabilizer $G_{0}^{i}$ of $C_{0}^{i}$ fixes an edge of $\hat{T}$. Since $\hat{T}$ resolves $T, G_{0}^{i}$ fixes an edge of $T$ as well.

Theorem 7.2 Let $S$ be a cocompact $G$-tree with finitely generated edge stabilizers and with $G$ finitely generated. Suppose that $G$ splits over a finite group. Then, $S$ may be iteratively $I^{-}, I I-$, and $I I I-$ simplified and then blown up to a $G$-tree $S^{\prime}$ such that the decomposition of $G$ given by edges of $S^{\prime}$ with finite stabilizer is non-trivial. Further, the simplifications and blow ups are all over finite groups. In particular, all point stabilizers of $S^{\prime}$ are finitely generated.

Proof Choose $T$ to be a minimal $G$-tree with one orbit of edges and with finite edge stabilizers. If an edge stabilizer of $S$ is finite then we may set $S^{\prime}=S$ and we are done. We may assume then that the edges stabilizers of $S$ are infinite.

Since edge stabilizers of $S$ are finitely generated and since $G$ is finitely generated, for each $s \in S, G_{s}$ is finitely generated, see for example [8, Lemma 32]. The edge stabilizers of $T$ are finite and by assumption $G_{s}$ is infinite and so either $G_{s}$ is contained in a unique vertex stabilizer of $T$ or some element of $G_{s}$ acts hyperbolically on $T$. In particular, there is a unique minimal cocompact $G_{s}$-subtree $T_{s}$ of $T$. Therefore we may apply Lemma 7.1 to simplify $X_{S}(T)$ to obtain $X_{N} \rightarrow S_{N}$.

Blow up $X_{N} \rightarrow S_{N}$ to obtain $X^{\prime} \rightarrow S^{\prime}$. Since $\left(X_{N}\right)_{\hat{f}}$ is a point for each edge $\hat{f}$ of $\hat{T}, S^{\prime}$ resolves $\hat{T}$. By Proposition [5.6, $\hat{T}$ is minimal and so $S^{\prime}$ is non-trivial.

Corollary 7.3 (Generalized Shenitzer-Swarup) Let $S$ be a minimal $G$-tree with finitely generated edge stabilizers. Suppose that $G$ splits over $\mathbf{1}$. Then, $S$ may be iteratively 1 -simplified to a tree $S^{\prime}$ such that the decomposition of $G$ determined by the edges of $S^{\prime}$ with trivial stabilizer is non-trivial.

The focus of this paper is on splittings over $\mathbf{1}$, ie, on free decompositions. In a future paper, we plan to explore splittings over small groups. Here is a 
sample analogue of Lemma 7.1 in that setting. Again, we use the notation of Example 5.5 .

Theorem 7.4 Suppose that $G$ is a freely indecomposable group. Suppose that $S$ and $T$ are $G$-trees such that

(1) $\bar{S}$ is compact;

(2) for each $s \in S$, there is a unique minimal cocompact $G_{s}$-subtree $T_{s}$ of $T$; and

(3) edge stabilizers of $T$ are infinite cyclic and $T$ has one orbit of edges.

Then, $X_{S}(T) \rightarrow S$ may be iteratively simplified to $X^{\prime} \rightarrow S^{\prime}$ where, for each edge $\hat{f}$ of $\hat{T}, X_{\hat{f}}^{\prime}$ is either a point with infinite cyclic stabilizer or a line with infinite cyclic stabilizer with generator acting by a non-trivial translation. Further, these simplifications are over $\mathbf{1}$ or $\mathbb{Z}$.

Proof Since $\hat{T}$ resolves $T, \hat{T}$ is minimal, and $G$ is freely indecomposable, it follows that the edge stabilizers of $\hat{T}$ are infinite cyclic. Thus, for $\hat{f}$ an edge

of $\hat{T},\left(X_{S}(T)\right)_{\hat{f}}$ is a $\mathbb{Z}$-tree. If this tree is not a point or a line, then it has a valence one vertex and a simplification is possible. Iterate.

\section{Algebraic consequences}

This section will be needed for algorithmic questions. We use the notation of Section 6. The goal is to describe the effect of simplifying on edge and vertex stabilizers.

Definition 8.1 Let $S$ be a $G$-tree and let $X \rightarrow S$ be a model with a cube $C$ with face $C_{0}$ such that $X^{\prime} \rightarrow S^{\prime}$ is the result of III-simplifying $C$ from $C_{0}$. Further, let $e$ be the image in $S$ of $C$, let $s_{0}$ be the image of $C_{0}$, and let $s_{e} \in \grave{e}$. Set $C_{s_{e}}=X_{s_{e}} \cap C$. Denote by $\bar{C}_{s_{e}}$ the image of $C_{s_{e}}$ in $\bar{X}_{s_{e}}$ and by $\bar{C}_{0}$ the image of $C_{0}$ in $\bar{X}_{s_{0}}$. There are three cases.

(1) $\bar{C}_{s_{e}}$ separates $\bar{X}_{s_{e}}$ and $\bar{C}_{0}$ separates $\bar{X}_{s_{0}}$.

(2) $\bar{C}_{s_{e}}$ separates $\bar{X}_{s_{e}}$, but $\bar{C}_{0}$ does not separate $\bar{X}_{s_{0}}$.

(3) $\bar{C}_{s_{e}}$ does not separate $\bar{X}_{s_{e}}$ and $\bar{C}_{0}$ does not separate $\bar{X}_{s_{0}}$.

Let $G_{0}$ be the stabilizer in $X$ of $C_{0}$. In Case (11), we say the simplification is a $G_{0}$-cleaving, in Case (2) a $G_{0}$-unkilling, and in Case (3) a $G_{0}$-unpulling. 
For the moment, we forget models and make some purely algebraic definitions. Here $G_{0}$ is a subgroup of the group $G$ and $\overrightarrow{\mathcal{H}}$ is a sequence of conjugacy classes of subgroups of $G$.

Definition 8.2 If there are subgroups $G^{\prime} \subset G$ and $G^{\prime \prime} \subset G$ containing $G_{0}$ such that

- $G=G^{\prime} *_{G_{0}} G^{\prime \prime}$, ie, the natural map $G^{\prime} *_{G_{0}} G^{\prime \prime} \rightarrow G$ is an isomorphism;

- some $\mathcal{H}_{0} \in \overrightarrow{\mathcal{H}}$ has a representative $H_{0} \in \mathcal{H}_{0}$ with subgroups $H_{0}^{\prime} \subset H_{0}$ and $H_{0}^{\prime \prime} \subset H_{0}$ satisfying

$-G_{0} \subset H_{0}^{\prime} \subset G^{\prime}$

- $G_{0} \subset H_{0}^{\prime \prime} \subset G^{\prime \prime} ;$ and

- $H_{0}=H_{0}^{\prime} *_{G_{0}} H_{0}^{\prime \prime} ;$ and

- for all $\mathcal{H} \neq \mathcal{H}_{0}$ in $\overrightarrow{\mathcal{H}}$, there is $H \in \mathcal{H}$ such that either $H \subset G^{\prime}$ or $H \subset G^{\prime \prime}$ then we say that $\overrightarrow{\mathcal{H}}$ can be $G_{0}$-cleaved in $G$.

Definition 8.3 If there is a subgroup $G^{\prime} \subset G$ containing $G_{0}$, a monomorphism $h: G_{0} \rightarrow G^{\prime}$, and $t \in G$ such that

- $G=G^{\prime} *_{h}=\left\langle G^{\prime}, t \mid t g t^{-1}=h(g), g \in G_{0}\right\rangle$;

- some $\mathcal{H}_{0} \in \overrightarrow{\mathcal{H}}$ has a representative $H_{0} \in \mathcal{H}_{0}$ with subgroups $H_{0}^{\prime}$ and $H_{0}^{\prime \prime}$ satisfying

- $G_{0} \subset H_{0}^{\prime} \subset G^{\prime}$

- $h\left(G_{0}\right) \subset H_{0}^{\prime \prime} \subset G^{\prime} ;$ and

- $H_{0}=H_{0}^{\prime} *_{G_{0}} t^{-1} H_{0}^{\prime \prime} t$; and

- for all $\mathcal{H} \neq \mathcal{H}_{0}$ in $\overrightarrow{\mathcal{H}}$ there is $H \in \mathcal{H}$ with $H \subset G^{\prime}$

then we say that $\overrightarrow{\mathcal{H}}$ can be $G_{0}$-unkilled in $G$.

Definition 8.4 If there is a subgroup $G^{\prime} \subset G$ containing $G_{0}$, a monomorphism $h: G_{0} \rightarrow G^{\prime}$, and $t \in G$ such that

- $G=G^{\prime} *_{h}$;

- some $\mathcal{H}_{0} \in \overrightarrow{\mathcal{H}}$ has a representative $H_{0} \in \mathcal{H}_{0}$ with a subgroup $H_{0}^{\prime}$ satisfying

$-t \in H_{0}$

- $H_{0}^{\prime} \subset G^{\prime}$

$-G_{0} \subset H_{0}^{\prime}$ 


$$
\begin{aligned}
& \text { - } h\left(G_{0}\right) \subset H_{0}^{\prime} \text {; and } \\
& \text { - } H_{0}=H_{0}^{\prime} *_{h} \text {; and }
\end{aligned}
$$

- for all $\mathcal{H} \neq \mathcal{H}_{0}$, there is $H \in \mathcal{H}$ such that $H \subset G^{\prime}$

then we say that $\overrightarrow{\mathcal{H}}$ can be $G_{0}$-unpulled in $G$.

If $\overrightarrow{\mathcal{H}}$ can be $G_{0}$-cleaved, $G_{0}$-unkilled, or $G_{0}$-unpulled then we say it can be III-G $G_{0}$-simplified.

Recall that if $s$ is a vertex of the $G$-tree $S$ then $\overrightarrow{\mathcal{H}}(s)$ denotes the sequence of conjugacy classes of subgroups of the stabilizer of $s$ represented by the stabilizers of edges incident to $s$. The sequence is indexed by the oriented edges $\bar{S}=S / G$ that are incident to the image in $\bar{S}$ of $s$.

Lemma 8.5 Let $S$ be a $G$-tree. If $S$ can be $G_{0}$-cleaved, $G_{0}$-unkilled, or $G_{0}$-unpulled then there is a vertex $s$ of $S$ such that $\overrightarrow{\mathcal{H}}(s)$ can be $G_{0}$-cleaved, $G_{0}$-unkilled, or $G_{0}$-unpulled in $G_{s}$.

Proof Assume that $S$ can be III- $G_{0}$-simplified. Let $X \rightarrow S$ be a model with a cube $C=I \times I$ and face $C_{0}=\{0\} \times I$ such that III-simplifying $C$ from $C_{0}$ produces $X^{\prime} \rightarrow S^{\prime}$. In particular, $G_{0}$ is the stabilizer of $C_{0}$. Let $s_{0}$ be the image of $C_{0}$ in $S$, let $e$ be the image of $C$ in $S$, and let $H_{0}$ be the stabilizer

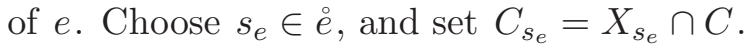

The desired splitting of $G_{s_{0}}$ is obtained by collapsing all edges of the $G_{s_{0}}$ tree $X_{s_{0}}$ that are not in the orbit of $C_{0}$. The desired $H_{0}$-tree is obtained by collapsing all edges of the $G_{0}$-tree $X_{s_{e}}$ that are not in the orbit of $C_{s_{e}}$. Thus, $\overrightarrow{\mathcal{H}}(s)$ can be III- $G_{0}$-simplified.

Definition 8.6 Suppose that $G$ is a group and that $\overrightarrow{\mathcal{H}}$ is a sequence of conjugacy classes of subgroups of $G$. Suppose that $G=G^{\prime} *_{G_{0}} G^{\prime \prime}$ or $G=G^{\prime} *_{G_{0}}$ and that, for all $\mathcal{H} \in \mathcal{H}, \mathcal{H}$ is conjugate into $G^{\prime}$ or $G^{\prime \prime}$. Then we say that $\overrightarrow{\mathcal{H}}$ can be $G_{0}$-blown up in $G$.

The proofs of the Lemmas 8.7 and 8.8 are very similar to that of Lemma 8.5 and are not provided.

Lemma 8.7 Let $G$ be a group and let $S$ be a $G$-tree. If $S$ can be $G_{0}$-blown up then there is a vertex $s$ of $S$ such that $\overrightarrow{\mathcal{H}}(s)$ can be $G_{0}$-blown up in $G_{s}$.

Lemma 8.8 Let $G$ be a group and let $S$ be a $G$-tree. If $S$ can be $G_{0}-I-$ simplified then $S$ has a valence one vertex with stabilizer $G_{0}$ whose incident edge has isomorphic stabilizer.

Remark 8.9 Recall that a II-simplification has no effect on $S$. 


\section{Algorithmic Results}

\subsection{More labeled graphs}

A map of labeled graphs $g: \Sigma_{1} \rightarrow \Sigma_{2}$ is a morphism if

- the induced map between universal covers is a morphism; and

- $g$ is label-preserving, ie, the following diagram commutes.

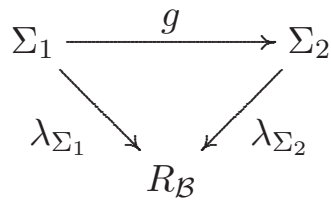

It is strict if this induced map is also strict. Stallings introduced labeled graphs into the study of free groups. The next lemma is key.

Lemma 9.1 (Stallings [27]) An immersion of labeled graphs induces an injection of fundamental groups.

An edge path in a labeled graph $\Sigma$ is a strict morphism $I \rightarrow \Sigma$ where $I$ is an oriented compact interval. If $I$ is a point then the edge path is trivial. A nontrivial edge path may be identified with a sequence of oriented edges $e_{0} \cdots e_{m}$ where, for $1 \leq i \leq m, \partial_{1} e_{i-1}=\partial_{0} e_{i}$. The product of edge paths $\sigma_{1}$ and $\sigma_{2}$ is denoted $\sigma_{1} \sigma_{2}$. An edge path is closed if the initial and terminal vertices of $I$ have the same image.

A loop in $\Sigma$ is a strict morphism $S \rightarrow \Sigma$ where $S$ is an oriented circle. A loop may be represented by a cyclic sequence of edges of $\Sigma$. An oriented edge of $\Sigma$ is crossed by a path or a loop if appears in the edge sequence representing the path or loop. The graph $\Sigma$ is tight if its labeling function $\lambda_{\Sigma}: \Sigma \rightarrow R_{\mathcal{B}}$ is an immersion. We record a simple corollary of Lemma 9.1

Corollary 9.2 If $g: I \rightarrow \Sigma$ is a tight non-trivial edge path then the element of $\pi_{1}\left(R_{\mathcal{B}}\right)$ represented by $\lambda_{\Sigma} \circ g$ is non-trivial. Equivalently, if $e_{0} \cdots e_{m}$ represents a tight non-trivial edge path and if the label of $e_{i}$ is $b_{i}^{\delta_{i}}\left(\delta_{i}= \pm 1\right)$ then $b_{0}^{\delta_{0}} \cdots b_{m}^{\delta_{m}}$ is non-trivial in $F_{\mathcal{B}}$. 
The Grushko decomposition of a finite graph of free groups

1863

\subsection{Operations on graphs}

\subsubsection{Coring}

A core graph is a graph such that every edge is crossed by an immersed loop. By Zorn's lemma, every graph $\Sigma$ has a unique maximal core subgraph, its core, denoted core $(\Sigma)$. A core graph contains no valence 0 or 1 vertices. If $\Sigma$ is connected and has finite fundamental group, then $\operatorname{core}(\Sigma)$ is finite. The core of a tree is empty. If $\Sigma$ is labeled, then so is core $(\Sigma)$. In fact, core is a functor from the category of labeled graphs and immersions to the category of labeled core graphs and immersions. The map core $(\Sigma) \subset \Sigma$ is natural with respect to this functor. The conjugacy class $[[H]]$ of a subgroup of $F_{\mathcal{B}}$ is uniquely represented by the core $\Sigma(H)$ of the cover $R_{\mathcal{B}, H}$ of $R_{\mathcal{B}}$ corresponding to $H$. The simple proof of the next lemma is left to the reader.

Lemma 9.3 Let $e$ be an edge of the labeled graph $\Sigma$.

- Suppose that e does not separate $\Sigma$. Then, $e \subset \operatorname{core}(\Sigma)$ if and only if there is an immersed loop crossing e exactly once.

- Suppose that e separates $\Sigma$. Then, $e \subset \operatorname{core}(\Sigma)$ if and only if there is an immersed loop crossing each of $e$ and $e^{-1}$ exactly once.

\subsubsection{Folds and tightening}

A morphism $g: \Sigma_{1} \rightarrow \Sigma_{2}$ of graphs is a fold if the induced map between universal covers is a fold. A fold induces a surjection on the level of fundamental groups. It is a homotopy equivalence unless the edges that are identified share both initial and terminal vertices [10].

A finite graph $\Sigma$ may be iteratively folded until it is tight. If $\Sigma$ is not finite, then the direct limit of the system of finite sequences of folds is well-defined. The result is the tightening of $\Sigma$ and is denoted $\operatorname{tight}(\Sigma)$. Fix a base vertex for $\Sigma$ (if $\Sigma$ is non-empty) and let $H$ denote the image $\left(\lambda_{\Sigma}\right)_{\#}\left(\pi_{1}(\Sigma)\right) \subset \pi_{1}\left(R_{\mathcal{B}}\right)$. Then, $\lambda_{\Sigma}$ lifts to $\Sigma \rightarrow R_{\mathcal{B}, H}$. The graph tight $(\Sigma)$ may be identified with the image of this lift. In fact, tight is a functor from the category of labeled graphs and strict morphisms to the category of tight labeled graphs and immersions. The quotient map $\Sigma \rightarrow \operatorname{tight}(\Sigma)$ is natural with respect to this functor. More generally, if $b \in \mathcal{B}$ and if $\Sigma$ is a labeled graph, then we define $\operatorname{tight}_{b}(\Sigma)$ as above except that only edges labeled $b$ or $b^{-1}$ are folded. 


\subsubsection{Applying an automorphism}

If $\Sigma$ is a labeled graph and $\alpha \in \operatorname{Aut}\left(F_{\mathcal{B}}\right)$, then $\alpha \Sigma$ is the labeled graph obtained by replacing each labeled oriented edge $e$ of $\Sigma$ by the sequence of labeled oriented edges $\alpha e$. More precisely, if the oriented edge $e$ has the label $b$ and if $\alpha(b)=w$ where $w$ is a reduced word of length $k$ in $\mathcal{B}$, then $\alpha e$ is obtained from $e$ by subdividing $e$ into $k$ subedges. The $i^{\text {th }}$ letter of $w$ has the form $c^{\delta}$ where $c \in \mathcal{B}$, and $\delta= \pm 1$. The $i^{t h}$ subedge of $e$ is given the label $c$ and an orientation agreeing with that of $e$ if $\delta$ is positive and the opposite orientation otherwise. The operation of applying the automorphism $\alpha$ is a functor from the category of labeled graphs and morphisms to itself. The construction gives a cellular map $\alpha: \Sigma \rightarrow \alpha \Sigma$ that is well defined up to a homotopy rel vertices and that is natural (but not a morphism).

Lemma 9.4 If $\sigma$ is an immersed edge path in $\alpha \Sigma$, then there are an immersed edge path $\hat{\sigma}$ in $\Sigma$ represented by $e_{0} \cdots e_{m}$, an initial edge subpath $\sigma_{0}$ of $\alpha\left(e_{0}\right)$, and a terminal edge subpath $\sigma_{m}$ of $\alpha\left(e_{m}\right)$ such that

(1) $\sigma_{0} \neq \alpha\left(e_{0}\right)$;

(2) $\sigma_{m} \neq \alpha\left(e_{m}\right)$; and

(3) $\alpha(\hat{\sigma})$ is the immersed edge path $\sigma_{0} \sigma \sigma_{m}$.

Proof We may view $\alpha \Sigma$ as being obtained from $\Sigma$ by subdividing and relabeling. With this in mind, any immersed edge path $\sigma$ in $\alpha \Sigma$ gives an immersed path $\sigma^{\prime}$ in $\Sigma$ that may not have endpoints vertices. This path extends uniquely to an immersed edge path $\hat{\sigma}$ that is minimal with respect to containing $\sigma^{\prime}$.

\subsubsection{Collapsing edges}

If $e$ is an edge of the labeled graph $\Sigma$, then $g: \Sigma \rightarrow \Sigma^{\prime}$ is a collapse of $e$ if the induced map between universal covers is the morphism collapsing a lift of $e$. In this case, we denote $\Sigma^{\prime}$ by collapse $e_{e}(\Sigma)$. More generally, if $\mathcal{E}$ is a set of edges in $\Sigma$ then we may collapse each edge in $\mathcal{E}$ to a point and obtain collapse $\mathcal{E}_{\mathcal{E}}(\Sigma)$. If $g: \Sigma \rightarrow \Sigma^{\prime}$ is a morphism, and if $\mathcal{E}^{\prime}$ is a set of edges in $\Sigma^{\prime}$, then there is an induced morphism collapse $\mathcal{E}^{\prime}(g):$ collapse $_{g^{-1}\left(\mathcal{E}^{\prime}\right)}(\Sigma) \rightarrow$ collapse $_{\mathcal{E}^{\prime}}\left(\Sigma^{\prime}\right)$. To each edge $e^{\prime}$ in collapse $\mathcal{E}_{\mathcal{E}}(\Sigma)$, we may associate the unique edge $e$ of $\Sigma$ such that collapse $_{\mathcal{E}}(e)=e^{\prime}$. The proof of the next lemma is left to the reader.

Lemma 9.5 (See [10]) (1) The quotient map $\Sigma \rightarrow$ collapse $_{\mathcal{E}}(\Sigma)$ induces a surjection of fundamental groups. 
(2) If $e^{\prime} \subset$ core $\left(\operatorname{collapse}_{\mathcal{E}}(\Sigma)\right)$ then $e \subset \operatorname{core}(\Sigma)$.

Remark 9.6 If $\mathcal{E}$ is the set of edges labeled $b$, then the operations tight ${ }_{b}$ and collapse $_{\mathcal{E}}$ commute.

\subsubsection{Operations on tight labeled core graphs}

If $\Sigma$ is a tight labeled core graph and if $\alpha \in \operatorname{Aut}\left(F_{\mathcal{B}}\right)$, then $\alpha_{\#} \Sigma$ is the labeled core graph core $(\operatorname{tight}(\alpha \Sigma))$ obtained by coring the tightening of $\alpha \Sigma$. If $\Sigma$ represents $[[H]]$, then $\alpha_{\#} \Sigma$ represents $[[\alpha H]]$.

\subsubsection{Sequences}

All the above notions extend to sequences of labeled graphs. For example, if $\vec{\Sigma}=\left\{\Sigma_{k}\right\}$ is a sequence of labeled graphs, then a path in $\vec{\Sigma}$ is a path $I \rightarrow G_{k_{0}}$ for some choice $k_{0}$ of $k, \alpha_{\#} \vec{\Sigma}$ denotes $\left\{\alpha_{\#} \Sigma_{k}\right\}$, etc.

A sequence $\overrightarrow{\mathcal{H}}$ of conjugacy classes of subgroups of $F_{\mathcal{B}}$ is uniquely represented by $\Sigma(\overrightarrow{\mathcal{H}})$. In the following definitions, $\vec{\Sigma}$ is a sequence of labeled graphs. For a labeled graph $\Sigma,|\Sigma|_{b}$ is the number of oriented edges of $\Sigma$ with label $b$. If $\vec{\Sigma}=\left\{\Sigma_{i}\right\}$ is a sequence of labeled graphs then $|\vec{\Sigma}|_{b}$ is the sum of the $\left|\Sigma_{i}\right|_{b}$.

\subsection{Elementary Whitehead automorphisms}

A reference for this section is [18. An extended permutation of $F_{\mathcal{B}}$ is an automorphism of $F_{\mathcal{B}}$ induced by a permutation of $\mathcal{B}^{ \pm 1}$. An elementary Whitehead automorphism is an automorphism $\alpha$ of $F_{\mathcal{B}}$ that is either an extended permutation or has the following form. There is an element $b \in \mathcal{B}^{ \pm 1}$ and a subset $A$ of $\mathcal{B}^{ \pm 1} \backslash\left\{b^{ \pm 1}\right\}$ such that

- if $c \in A \backslash A^{-1}$ then $\alpha(c)=b c$;

- if $c \in A \cap A^{-1}$ then $\alpha(c)=b c b^{-1}$; and

- if $c \notin A \cup A^{-1}$ then $\alpha(c)=c$.

We call $b$ the distinguished label of $\alpha$.

Remark 9.7 Let $\vec{\Sigma}$ be a sequence of labeled graphs and let $\alpha$ be an elementary Whitehead automorphism with distinguished label $b$. There is a $1-1$ correspondence between the set of edges of $\vec{\Sigma}$ not labeled $b$ and the edges of 
$\alpha \vec{\Sigma}$ not labeled $b$. In $\alpha \vec{\Sigma}$, there are old and new edges labeled $b$. The terminal vertex of each new edge has valence 2 and the other incident edge is not labeled $b$. Such a valence 2 vertex is new; other vertices are old. The subgraph of $\alpha \vec{\Sigma}$ consisting of new edges is a forest each component of which is a cone over a set of new vertices with base an old vertex. All edges of the cone have initial vertex the base. See Figure 15.
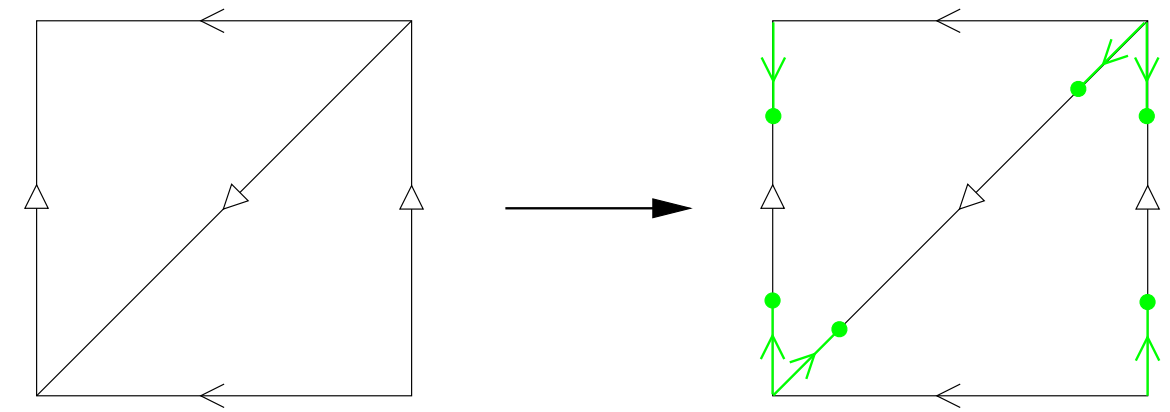

Figure 15: $\alpha(a)=a, \alpha(b)=a b a^{-1}$

Remark 9.8 For $\alpha \in \operatorname{Aut}\left(F_{\mathcal{B}}\right)$, the sequence of folds needed to tighten $\alpha R_{\mathcal{B}}$ algorithmically gives a factorization of $\alpha$ as a product of elementary Whitehead automorphisms.

The next lemma is a consequence of Step 1 of the proof of the proposition on page 455 of [4].

Lemma 9.9 Let $g: \Sigma_{0} \rightarrow \Sigma_{1}$ be a strict morphism of labeled graphs that is surjective on the level of fundamental groups. Then, there is a fold $g^{\prime}: \Sigma_{0} \rightarrow \Sigma^{\prime}$ such that $g$ factors as

$$
\Sigma_{0} \stackrel{g^{\prime}}{\rightarrow} \Sigma^{\prime} \rightarrow \Sigma_{1}
$$

Lemma 9.10 Let $g: \Sigma_{0} \rightarrow \Sigma_{1}$ be a strict morphism of labeled graphs that is surjective on the level of fundamental groups. Suppose that, for some $b \in \mathcal{B}$, $\left|\Sigma_{1}\right|_{b}<\left|\Sigma_{0}\right|_{b}$. Then, there are strict morphisms making the following diagram commute

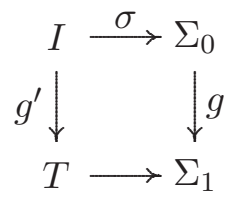

where 
- where $\sigma$ is an immersed edge path represented by $e_{0} e_{1} \cdots e_{m}$;

- $T$ is a labeled tree;

- $e_{0}$ and $e_{m}^{-1}$ are labeled by $b^{\prime}=b$ or $b^{-1}$;

- for $0<i<m, e_{i}$ is not labeled by $b$ or $b^{-1}$; and

- $g^{\prime}\left(e_{0}\right)=g^{\prime}\left(e_{m}^{-1}\right)$.

Proof Since $\left|\Sigma_{1}\right|_{b}<\left|\Sigma_{0}\right|_{b}$ there are distinct edges $e$ and $e^{\prime}$ in $\Sigma_{0}$ each labeled $b$ that are identified under $g$. Consider the lift $\tilde{g}: \tilde{\Sigma}_{0} \rightarrow \tilde{\Sigma}_{1}$ to universal covers. Because $g$ induces a surjection on the level of fundamental groups, there are lifts $\tilde{e}$ and $\tilde{e}^{\prime}$ of $e$ and $e^{\prime}$ to $\tilde{\Sigma}_{0}$ that are identified under $\tilde{g}$. Choose $\tilde{e}$ and $\tilde{e}^{\prime}$ with this property so that the subtree $I$ they span has minimal diameter (with respect to the edge metric). The edge path $\sigma: I \rightarrow \Sigma_{0}$ is the restriction of the first covering projection. The edge path $\sigma$ factors as $I \rightarrow T=\tilde{g}(I) \rightarrow \Sigma_{1}$ where the first factor is induced by the restriction of $\tilde{g}$ to $I$ and the second factor is the restriction of the second covering projection.

Lemma 9.11 Let $\vec{\Sigma}$ be a sequence of tight labeled graphs and let $\alpha \in \operatorname{Aut}\left(F_{\mathcal{B}}\right)$ be an elementary Whitehead automorphism with distinguished label $b$. Let $\mathcal{E}$ (respectively $\mathcal{E}^{\prime}$ ) be the set of edges of $\Sigma$ (respectively tight $(\alpha \Sigma)$ ) that are labeled $b$. Then, the following diagram commutes and the lower horizontal arrow is an isomorphism.

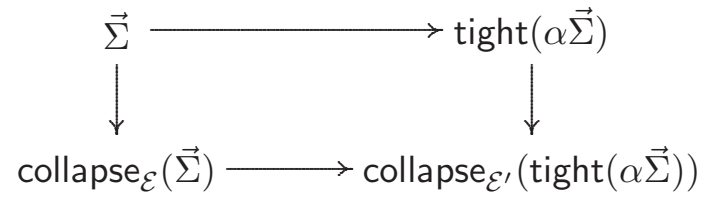

In particular, there is a natural 1-1 correspondence between edges of $\vec{\Sigma}$ not labeled $b^{ \pm 1}$ and edges of $\operatorname{tight}(\alpha \vec{\Sigma})$ not labeled $b^{ \pm 1}$.

Proof Let $\mathcal{E}^{\prime \prime}$ be the set of edges of $\alpha \Sigma$ that are labeled $b$. We have a commuting diagram

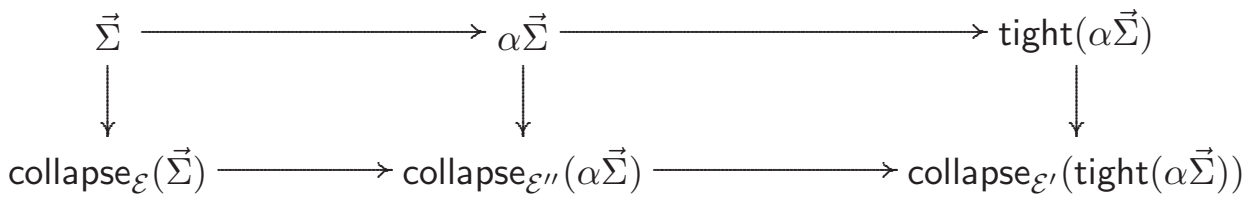

It is clear that the lower left horizontal arrow is an isomorphism and that the lower right arrow is strict and surjective. 
In order to obtain a contradiction, assume

$$
\text { collapse }_{\mathcal{E}^{\prime \prime}}(\alpha \vec{\Sigma}) \rightarrow \text { collapse }_{\mathcal{E}^{\prime}}(\operatorname{tight}(\alpha \vec{\Sigma}))
$$

is not injective. Since this map is $\pi_{1}$-surjective, by Lemma 9.9 there are two edges not labeled $b$ or $b^{-1}$ with the same image. It follows that there are two edges not labeled $b$ or $b^{-1}$ with the same image under $\alpha \vec{\Sigma} \rightarrow \operatorname{tight}(\alpha \vec{\Sigma})$. Using Lemma 9.10 and taking a subpath if necessary, there is an immersed edge path $\sigma: I \rightarrow \alpha \vec{\Sigma}$ represented by $e_{0} e_{1} \cdots e_{m}$ such that

- the label of $e_{0}$ and $e_{m}^{-1}$ is $b^{\prime} \neq b^{ \pm 1}$;

- the label of $e_{i}$ is $b^{ \pm 1}$ for all $0<i<m$; and

- $I \rightarrow \alpha \vec{\Sigma} \rightarrow \operatorname{tight}(\alpha \vec{\Sigma})$ factors through a tree.

If $\hat{\sigma}$ is the immersed edge path in $\vec{\Sigma}$ determined by $\sigma$ as in Lemma 9.4 then

- the label of $\hat{e}_{0}$ and $\hat{e}_{\hat{m}}^{-1}$ is $b^{\prime}$; and

- $\hat{e}_{i}$ is labeled $b^{ \pm 1}$ for $1<i<\hat{m}$.

Since $\hat{\sigma}$ is an immersion, all the $\hat{e}_{i}, 1<i<\hat{m}$, are consistently oriented. It is easy to see then that $I \rightarrow \alpha \vec{\Sigma} \rightarrow \operatorname{tight}(\alpha \vec{\Sigma})$ cannot factor through a tree, contradiction.

Lemma 9.12 Let $\vec{\Sigma}$ be a sequence of tight labeled core graphs and let $\alpha$ be an elementary Whitehead automorphism with distinguished label $b$, let $e$ be an edge of $\vec{\Sigma}$ not labeled $b^{ \pm 1}$, and let $e^{\prime}$ be the corresponding edge in $\operatorname{tight}(\alpha \vec{\Sigma})$. Then, $e^{\prime}$ is in $\alpha_{\#} \vec{\Sigma}=\operatorname{core}(\operatorname{tight}(\alpha \vec{\Sigma}))$. In particular, there is a natural 1-1 correspondence between edges of $\vec{\Sigma}$ not labeled $b^{ \pm 1}$ and edges of $\alpha_{\#} \vec{\Sigma}$ not labeled $b^{ \pm 1}$.

Proof Suppose that $e$ separates (respectively does not separate) its component. By Lemma 9.3, there is an immersed loop $g: S \rightarrow \vec{\Sigma}$ crossing $e$ (respectively crossing $e^{\prime}$ and $e^{-1}$ each) exactly once. It follows from Lemma 9.11 that the immersed loop tight $(\alpha g)$ crosses $e^{\prime}$ (respectively $e^{\prime}$ and $e^{\prime-1}$ each) exactly once. By Lemma 9.3 $e^{\prime}$ is contained in $\alpha_{\#} \vec{\Sigma}$.

\subsection{Complexity}

If $\overrightarrow{\mathcal{H}}$ is a finite sequence of conjugacy classes of finitely generated subgroups of $F_{\mathcal{B}}$ and if $b \in \mathcal{B}$, then $|\overrightarrow{\mathcal{H}}|_{b}$ is the number of edges in $\Sigma(\overrightarrow{\mathcal{H}})$ that are labeled with $b$. The complexity of $\overrightarrow{\mathcal{H}}$, denoted $c(\overrightarrow{\mathcal{H}})$, is the number of edges in $\Sigma(\overrightarrow{\mathcal{H}})$ or equivalently $\sum_{b \in \mathcal{B}}|\overrightarrow{\mathcal{H}}|_{b}$. 
We will also need a finer measure of complexity of $\overrightarrow{\mathcal{H}}$. Define the lexity of $\overrightarrow{\mathcal{H}}$, denoted lex $(\overrightarrow{\mathcal{H}})$, to be the sequence of non-negative integers $\left\{|\Sigma(\overrightarrow{\mathcal{H}})|_{b}\right\}_{b \in \mathcal{B}}$ arranged in non-decreasing order. The set $\mathcal{L}$ of non-decreasing sequences of non-negative integers is well-ordered lexicographically. Let $\operatorname{minlex}(\overrightarrow{\mathcal{H}})$ denote $\min _{b \in \mathcal{B}}\left\{|\Sigma(\overrightarrow{\mathcal{H}})|_{b}\right\}$.

Lemma 9.13 Let $\overrightarrow{\mathcal{H}}$ be a finite sequence of conjugacy classes of finitely generated subgroups of $F_{\mathcal{B}}$ and let $\alpha$ be an elementary Whitehead automorphism. Then $\mathrm{c}(\alpha \overrightarrow{\mathcal{H}})<\mathrm{c}(\overrightarrow{\mathcal{H}})$ if and only if $\operatorname{lex}(\alpha \overrightarrow{\mathcal{H}})<\operatorname{lex}(\overrightarrow{\mathcal{H}})$. If further $\alpha$ has distinguished label $b$, then $\operatorname{lex}(\alpha \overrightarrow{\mathcal{H}}) \leq \operatorname{lex}(\overrightarrow{\mathcal{H}})$ if and only if $|\alpha \overrightarrow{\mathcal{H}}|_{b} \leq|\overrightarrow{\mathcal{H}}|_{b}$ with equality if and only if $|\alpha \overrightarrow{\mathcal{H}}|_{b}=|\overrightarrow{\mathcal{H}}|_{b}$.

Proof Since extended permutations preserve both c and lex, we may suppose that $\alpha$ has distinguished label $b \in \mathcal{B}$. It follows from Lemma 9.12 that if $b^{\prime} \neq b^{ \pm 1}$, then the number of times that $b^{\prime}$ appears in $\Sigma(\alpha \overrightarrow{\mathcal{H}})$ is the same as the number of times that $b^{\prime}$ appears in $\Sigma(\overrightarrow{\mathcal{H}})$.

\subsection{Gersten's Theorem}

Let $\overrightarrow{\mathcal{H}}$ be a finite sequence of conjugacy classes of finitely generated subgroups of $F_{\mathcal{B}}$. If $\mathrm{c}(\overrightarrow{\mathcal{H}})=\min \left\{\mathrm{c}(\alpha \overrightarrow{\mathcal{H}}) \mid \alpha \in \operatorname{Aut}\left(F_{\mathcal{B}}\right)\right\}$ then $\overrightarrow{\mathcal{H}}$ is a Gersten representative for the orbit $\operatorname{Aut}\left(F_{\mathcal{B}}\right) \overrightarrow{\mathcal{H}}$. We also write that $\overrightarrow{\mathcal{H}}$ is a Gersten representative for any element of the orbit. Since $\overrightarrow{\mathcal{H}}$ is an element of this orbit, we often simply write that $\overrightarrow{\mathcal{H}}$ is a Gersten representative. A finite set of generators for a representative $H \in \mathcal{H}$ for each $\mathcal{H} \in \overrightarrow{\mathcal{H}}$ is a finite generating system for $\overrightarrow{\mathcal{H}}$. S M Gersten [14] 18 gave an algorithm that when input a finite generating system for $\overrightarrow{\mathcal{H}}$ outputs the finite set of Gersten representatives for $\overrightarrow{\mathcal{H}}$.

\section{Theorem 9.14 14, 18,}

(1) If $\overrightarrow{\mathcal{H}}$ is a finite sequence of conjugacy classes of finitely generated subgroups of $F_{\mathcal{B}}$ that is not a Gersten representative, then there is an elementary Whitehead automorphism $\alpha$ such that $\mathrm{c}(\alpha \overrightarrow{\mathcal{H}})<\mathrm{c}(\overrightarrow{\mathcal{H}})$.

(2) If $\overrightarrow{\mathcal{H}}$ and $\overrightarrow{\mathcal{H}}^{\prime}$ are Gersten representatives for $\overrightarrow{\mathcal{H}}$, then there is a finite sequence $\left\{\alpha_{k}\right\}_{k=1}^{m}$ of elementary Whitehead automorphisms and a sequence

$$
\left\{\overrightarrow{\mathcal{H}}=\overrightarrow{\mathcal{H}}_{0}, \overrightarrow{\mathcal{H}}_{1}, \cdots, \overrightarrow{\mathcal{H}}_{m}=\overrightarrow{\mathcal{H}}^{\prime}\right\}
$$

of Gersten representatives such that $\alpha_{k} \overrightarrow{\mathcal{H}}_{k-1}=\overrightarrow{\mathcal{H}}_{k}$ for $1 \leq k \leq m$. 
Corollary 9.15 If $\overrightarrow{\mathcal{H}}$ is a finite sequence of conjugacy classes of finitely generated subgroups of $F_{\mathcal{B}}$, then there is an algorithm that when input a finite generating system for $\overrightarrow{\mathcal{H}}$ outputs a Gersten representative for $\overrightarrow{\mathcal{H}}$.

Proof Iteratively apply elementary Whitehead automorphisms to $\overrightarrow{\mathcal{H}}$ until complexity cannot be decreased. The resulting sequence is a Gersten representative by Theorem 9.14(1).

Corollary 9.16 Let $\overrightarrow{\mathcal{H}}$ be a finite sequence of conjugacy classes of finitely generated subgroups of $F_{\mathcal{B}}$. Then, there is an algorithm that when input a finite generating system for $\overrightarrow{\mathcal{H}}$ outputs the finitely many Gersten representatives of $\overrightarrow{\mathcal{H}}$.

Proof By Corollary 9.15 we may assume that $\overrightarrow{\mathcal{H}}$ is a Gersten representative. Consider the graph whose vertices are finite sequences of conjugacy classes of finitely generated subgroups of $F_{\mathcal{B}}$ of complexity equal to $\mathrm{c}(\overrightarrow{\mathcal{H}})$, and where two vertices $\overrightarrow{\mathcal{H}}_{1}$ and $\overrightarrow{\mathcal{H}}_{2}$ are connected by an edge if there is an elementary Whitehead automorphism $\alpha$ such that $\alpha \overrightarrow{\mathcal{H}}_{1}=\overrightarrow{\mathcal{H}}_{2}$. By Theorem 9.14(2), the component of this graph containing $\overrightarrow{\mathcal{H}}$ has vertices that are precisely the Gersten representatives of $\overrightarrow{\mathcal{H}}$.

\subsection{Consequences of Lemma 9.13}

In this section we show that simplifications can be detected using Gersten representatives. Throughout this section, $\overrightarrow{\mathcal{H}}$ is a finite sequence of conjugacy classes of finitely generated subgroups of $F_{\mathcal{B}}$.

Lemma 9.17 The following are equivalent.

(1) There is an $\alpha \in \operatorname{Aut}\left(F_{\mathcal{B}}\right)$ such that $\operatorname{minlex}(\alpha \overrightarrow{\mathcal{H}})=0$.

(2) For some (any) Gersten representative $\overrightarrow{\mathcal{H}}^{\prime}$ of $\overrightarrow{\mathcal{H}}$, $\operatorname{minlex}\left(\overrightarrow{\mathcal{H}}^{\prime}\right)=0$.

Proof By Lemma 9.13, for any $\alpha \in \operatorname{Aut}\left(F_{\mathcal{B}}\right)$ and any Gersten representative $\overrightarrow{\mathcal{H}}^{\prime}$ of $\overrightarrow{\mathcal{H}}, \operatorname{lex}(\alpha \overrightarrow{\mathcal{H}}) \geq \operatorname{lex}\left(\overrightarrow{\mathcal{H}}^{\prime}\right)$. Thus, (11) $\Longleftrightarrow$ (2).

Lemma 9.18 Let $\mathcal{B}^{\prime} \subset \mathcal{B}$ and suppose that $\overrightarrow{\mathcal{H}}^{\prime}$ is a finite sequence of conjugacy classes of finitely generated subgroups of $F_{\mathcal{B}^{\prime}}$. The following are equivalent.

(1) $\overrightarrow{\mathcal{H}}^{\prime}$ is a Gersten representative with respect to $\mathcal{B}^{\prime}$. 
(2) $\overrightarrow{\mathcal{H}}^{\prime}$ is a Gersten representative with respect to $\mathcal{B}$.

Proof That $(2) \Longrightarrow(1)$ is clear. Suppose (1), but not (2). By Lemma 9.13 there is then an elementary Whitehead automorphism $\alpha \in \operatorname{Aut}\left(F_{\mathcal{B}}\right)$ with distinguished label $b \notin\left(\mathcal{B}^{\prime}\right)^{ \pm 1}$ such that $\left|\alpha \overrightarrow{\mathcal{H}}^{\prime}\right|_{b}<\left|\overrightarrow{\mathcal{H}}^{\prime}\right|_{b}=0$, contradiction.

Recall that the terms visibly blown up, visibly unpulled, visibly unkilled, visibly uncleaved, and visibly simplified were defined in Definition 2.5.

Lemma 9.19 The following are equivalent.

(1) There is an $\alpha \in \operatorname{Aut}\left(F_{\mathcal{B}}\right)$ such that $\Sigma(\alpha \overrightarrow{\mathcal{H}})$ can be visibly blown up.

(2) For some (every) Gersten representative $\overrightarrow{\mathcal{H}}^{\prime}$ of $\overrightarrow{\mathcal{H}}, \Sigma\left(\overrightarrow{\mathcal{H}}^{\prime}\right)$ can be visibly blown up.

Proof The lemma will follow from:

Claim If $\Sigma(\overrightarrow{\mathcal{H}})$ can be visibly blown up and if $\alpha$ is an elementary Whitehead automorphism with $\operatorname{lex}(\alpha \overrightarrow{\mathcal{H}}) \leq \operatorname{lex}(\overrightarrow{\mathcal{H}})$ then either $\Sigma(\alpha \overrightarrow{\mathcal{H}})$ can be visibly blown up or there is an automorphism $\alpha^{\prime}$ with $\operatorname{lex}\left(\alpha^{\prime} \overrightarrow{\mathcal{H}}\right)<\operatorname{lex}(\overrightarrow{\mathcal{H}})$ such that $\Sigma\left(\alpha^{\prime} \overrightarrow{\mathcal{H}}\right)$ can be visibly blown up.

We now prove the claim. Suppose $\mathcal{B}=\mathcal{B}^{\prime} \sqcup \mathcal{B}^{\prime \prime}$ is a non-trivial partition such that each element of $\overrightarrow{\mathcal{H}}$ has a representative in either $F_{\mathcal{B}^{\prime}}$ or $F_{\mathcal{B}^{\prime \prime}}$. If $\alpha$ is an extended permutation, then the claim is clear.

Alternatively, let $b \in \mathcal{B}^{ \pm 1}$ be the distinguished label of $\alpha$ and suppose without loss that $b \in\left(\mathcal{B}^{\prime}\right)^{ \pm 1}$. Let $\alpha^{\prime}$ be the automorphism that agrees with $\alpha$ on $\mathcal{B}^{\prime}$ and that is the identity on $\mathcal{B}^{\prime \prime}$. It is clear that $\Sigma\left(\alpha^{\prime} \overrightarrow{\mathcal{H}}\right)$ can be visibly blown up. Now, $\Sigma(\alpha \overrightarrow{\mathcal{H}})$ can be visibly blown up if and only if, for each $\mathcal{H} \in \overrightarrow{\mathcal{H}}$ with a representative in $F_{\mathcal{B}^{\prime \prime}}, \alpha \mathcal{H}$ has a representative in $F_{\mathcal{B}^{\prime \prime}}$. By Lemma 9.13. this occurs if and only if $|\alpha \mathcal{H}|_{b}>0$ for such $\mathcal{H}$ and this occurs if and only if $\left|\alpha^{\prime} \overrightarrow{\mathcal{H}}\right|_{b}<|\alpha \overrightarrow{\mathcal{H}}|_{b}$.

Lemma 9.20 Suppose that for some (any) Gersten representative $\overrightarrow{\mathcal{H}}^{\prime}$ of $\overrightarrow{\mathcal{H}}$ we have $\operatorname{minlex}\left(\overrightarrow{\mathcal{H}}^{\prime}\right) \neq 0$. Then, the following are equivalent.

(1) There is an $\alpha \in \operatorname{Aut}\left(F_{\mathcal{B}}\right)$ such that $\min \operatorname{lex}(\alpha \overrightarrow{\mathcal{H}})=1$.

(2) For some (any) Gersten representative $\overrightarrow{\mathcal{H}}^{\prime}$ of $\overrightarrow{\mathcal{H}}$, $\operatorname{minlex}\left(\overrightarrow{\mathcal{H}}^{\prime}\right)=1$.

(3) $\overrightarrow{\mathcal{H}}$ can be either visibly unkilled or visibly unpulled. 
Proof In the presence of $\operatorname{minlex}\left(\overrightarrow{\mathcal{H}}^{\prime}\right) \neq 0$, (11) $\Longleftrightarrow$ (2) by Lemma 9.13, Suppose that (2) holds. There are two cases: there is a label $b \in \mathcal{B}$ that appears exactly once in $\Sigma\left(\overrightarrow{\mathcal{H}}^{\prime}\right)$ and (a) the edge labeled $b$ separates its component and (b) edge labeled $b$ does not separate its component. It is an easy exercise to show that in case (a) $\Sigma(\overrightarrow{\mathcal{H}})$ can be visibly unkilled and in case (b) $\Sigma(\overrightarrow{\mathcal{H}})$ can be visibly unpulled.

Suppose that (3) holds. Then there is free factorization $F_{\mathcal{B}}=F^{1} *\langle 1\rangle=F^{1} *\langle t\rangle$ as in Definition 8.3 or Definition 8.4. Let $\mathcal{B}^{1}$ be a basis for $F^{1}$. Choose an $\alpha \in \operatorname{Aut}\left(F_{\mathcal{B}}\right)$ so that $\alpha\left(\mathcal{B}^{1} \sqcup\{t\}\right)=\mathcal{B}$. Then $\operatorname{minlex}(\alpha \overrightarrow{\mathcal{H}})=1$.

The next lemma will be used to prove Lemma 9.23.

Lemma 9.21 Suppose that

(1) $\operatorname{minlex}(\overrightarrow{\mathcal{H}})>1$;

(2) $\mathcal{B}=\mathcal{B}^{\prime} \sqcup \mathcal{B}^{\prime \prime}$ is a non-trivial partition;

(3) $\Sigma(\overrightarrow{\mathcal{H}})=\Sigma\left(\overrightarrow{\mathcal{H}}^{\prime}\right) \vee \Sigma\left(\overrightarrow{\mathcal{H}}^{\prime \prime}\right)$ where $\overrightarrow{\mathcal{H}}^{\prime}$ is a Gersten representative in $F_{\mathcal{B}^{\prime}}$ and $\overrightarrow{\mathcal{H}}^{\prime \prime}$ is a Gersten representative in $F_{\mathcal{B}^{\prime \prime}}$; and

(4) $\alpha \in \operatorname{Aut}\left(F_{\mathcal{B}}\right)$ is an elementary Whitehead automorphism with distinguished label $b \in\left(\mathcal{B}^{\prime}\right)^{ \pm 1}$ such that $\operatorname{lex}(\alpha \overrightarrow{\mathcal{H}}) \leq \operatorname{lex}(\overrightarrow{\mathcal{H}})$

Then,

- $\alpha \overrightarrow{\mathcal{H}^{\prime \prime}}=\overrightarrow{\mathcal{H}}^{\prime \prime}$

- $\alpha \overrightarrow{\mathcal{H}}^{\prime}$ is a Gersten representative for $\overrightarrow{\mathcal{H}}^{\prime}$; and

- $\Sigma(\alpha \overrightarrow{\mathcal{H}})=\Sigma\left(\alpha \overrightarrow{\mathcal{H}}^{\prime}\right) \vee \Sigma\left(\overrightarrow{\mathcal{H}}^{\prime \prime}\right)$.

In particular, $\overrightarrow{\mathcal{H}}$ satisfying (1), (2), and (3) is a Gersten representative.

Proof Note:

- $\Sigma(\alpha \overrightarrow{\mathcal{H}})=\operatorname{tight}\left(\Sigma\left(\alpha \overrightarrow{\mathcal{H}}^{\prime}\right) \vee I \vee \Sigma\left(\alpha \overrightarrow{\mathcal{H}}^{\prime \prime}\right)\right)$ for some labeled graph $I$ homeomorphic to a compact interval. This follows because in tightening $\alpha \Sigma(\overrightarrow{\mathcal{H}})$, we can tighten $\alpha \Sigma\left(\overrightarrow{\mathcal{H}}^{\prime}\right)$ and $\alpha \Sigma\left(\overrightarrow{\mathcal{H}}^{\prime \prime}\right)$ first.

- The subgraph of $\Sigma\left(\alpha \overrightarrow{\mathcal{H}}^{\prime \prime}\right)$ consisting of edges labeled $b$ is a tree whose components are single (non-loop) edges. This follows from Remark 9.7 and the following commutative diagram where $\mathcal{E}$ is the set of edges of $\Sigma\left(\alpha \overrightarrow{\mathcal{H}^{\prime \prime}}\right)$ that are labeled $b$.

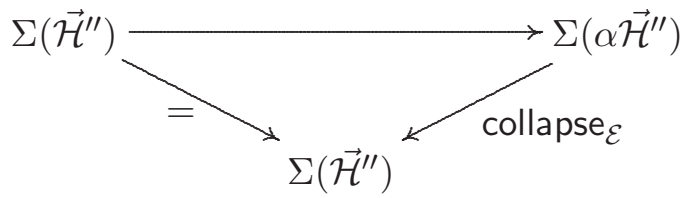


It follows that in tightening $\Sigma\left(\alpha \overrightarrow{\mathcal{H}^{\prime}}\right) \vee I \vee \Sigma\left(\alpha \overrightarrow{\mathcal{H}^{\prime \prime}}\right)$ at most one edge of $\Sigma\left(\alpha \overrightarrow{\mathcal{H}^{\prime}}\right)$ folds with an edge of $\Sigma\left(\alpha \overrightarrow{\mathcal{H}}^{\prime \prime}\right)$. Hence,

$$
\begin{aligned}
\mathrm{c}\left(\overrightarrow{\mathcal{H}}^{\prime}\right)+\mathrm{c}\left(\overrightarrow{\mathcal{H}}^{\prime \prime}\right) & =\mathrm{c}(\overrightarrow{\mathcal{H}}) \geq \mathrm{c}(\alpha \overrightarrow{\mathcal{H}}) \\
& \geq \mathrm{c}\left(\alpha \overrightarrow{\mathcal{H}}^{\prime}\right)+\mathrm{c}\left(\alpha \overrightarrow{\mathcal{H}}^{\prime \prime}\right)-1 \geq \mathrm{c}\left(\overrightarrow{\mathcal{H}}^{\prime}\right)+\mathrm{c}\left(\overrightarrow{\mathcal{H}}^{\prime \prime}\right)-1 .
\end{aligned}
$$

Thus, either $\mathrm{c}\left(\alpha \overrightarrow{\mathcal{H}}^{\prime \prime}\right)=\mathrm{c}\left(\overrightarrow{\mathcal{H}}^{\prime \prime}\right)$ or $\mathrm{c}\left(\alpha \overrightarrow{\mathcal{H}}^{\prime \prime}\right)=\mathrm{c}\left(\overrightarrow{\mathcal{H}}^{\prime \prime}\right)+1$. In the former case, we are done. The latter case cannot occur. Indeed, otherwise $\left|\alpha \overrightarrow{\mathcal{H}}^{\prime \prime}\right|_{b}=1$. But then, by Lemmas 9.18 and 9.20, $\operatorname{minlex}\left(\overrightarrow{\mathcal{H}}^{\prime \prime}\right) \leq 1$ and hence $\operatorname{minlex}(\overrightarrow{\mathcal{H}}) \leq 1$, contradiction.

Remark 9.22 Without (1), Lemma 9.21 is false. Consider $\mathcal{B}=\{a, b\} \sqcup\{c\}$, $H=\left\langle a b^{-1}, c\right\rangle$, and $\alpha(a)=a, \alpha(b)=b, \alpha(c)=b c$.

Lemma 9.23 Suppose that $\operatorname{minlex}\left(\overrightarrow{\mathcal{H}}^{\prime}\right)>1$ for some (any) Gersten representative $\overrightarrow{\mathcal{H}}^{\prime}$ of $\overrightarrow{\mathcal{H}}$. Then, the following are equivalent.

(1) There is $\alpha \in \operatorname{Aut}\left(F_{\mathcal{B}}\right)$ such that $\Sigma(\alpha \overrightarrow{\mathcal{H}})$ can be visibly cleaved.

(2) For some (every) Gersten representative $\overrightarrow{\mathcal{H}}^{\prime}$ of $\overrightarrow{\mathcal{H}}, \Sigma\left(\overrightarrow{\mathcal{H}}^{\prime}\right)$ can be visibly cleaved.

Proof (2) $\Longrightarrow$ (11) is clear. We now show (11) $\Longrightarrow$ (2). Suppose that $\Sigma(\alpha \overrightarrow{\mathcal{H}})=$ $\Sigma\left(\overrightarrow{\mathcal{H}}^{\prime}\right) \vee \Sigma\left(\overrightarrow{\mathcal{H}^{\prime \prime}}\right)$ where all labels of $\Sigma\left(\overrightarrow{\mathcal{H}}^{\prime}\right)$ are in $\mathcal{B}^{\prime \pm 1}$ and all labels of $\Sigma\left(\overrightarrow{\mathcal{H}}^{\prime \prime}\right)$ are in $\mathcal{B}^{\prime \prime \pm 1}$ for some non-trivial partition $\mathcal{B}=\mathcal{B}^{\prime} \sqcup \mathcal{B}^{\prime \prime}$. Choose $\alpha^{\prime} \in \operatorname{Aut}\left(F_{\mathcal{B}^{\prime}}\right)$ and $\alpha^{\prime \prime} \in \operatorname{Aut}\left(F_{\mathcal{B}^{\prime \prime}}\right)$ such that $\alpha^{\prime} \overrightarrow{\mathcal{H}}^{\prime}$ and $\alpha^{\prime \prime} \overrightarrow{\mathcal{H}}^{\prime \prime}$ are Gersten representatives. Let $\alpha \in \operatorname{Aut}\left(F_{\mathcal{B}}\right)$ agree with $\alpha^{\prime}$ on $F_{\mathcal{B}^{\prime}}$ and $\alpha^{\prime \prime}$ on $F_{\mathcal{B}^{\prime \prime}}$. Then, $\Sigma(\alpha \overrightarrow{\mathcal{H}})=$ $\Sigma\left(\alpha^{\prime} \overrightarrow{\mathcal{H}}^{\prime}\right) \vee I^{\prime} \vee I^{\prime \prime} \vee \Sigma\left(\alpha^{\prime \prime} \overrightarrow{\mathcal{H}}^{\prime \prime}\right)$ where $I^{\prime}$ and $I^{\prime \prime}$ are labeled graphs homeomorphic to compact intervals, all labels of $I^{\prime}$ are in $\left(\mathcal{B}^{\prime}\right)^{ \pm 1}$, and all labels of $I^{\prime \prime}$ are in $\left(\mathcal{B}^{\prime \prime}\right)^{ \pm 1}$. We will now show that $\Sigma\left(\alpha^{\prime} \overrightarrow{\mathcal{H}}^{\prime}\right) \vee \Sigma\left(\alpha^{\prime \prime} \overrightarrow{\mathcal{H}}^{\prime \prime}\right)$ is also a representative for $\overrightarrow{\mathcal{H}}$. Suppose that $I^{\prime \prime}$ is not trivial and that the edge $e$ of $I^{\prime \prime}$ with initial vertex in $\Sigma\left(\alpha^{\prime} \overrightarrow{\mathcal{H}}^{\prime}\right) \vee I^{\prime}$ is labeled $b$. Let $\alpha_{b}$ be the elementary Whitehead automorphism that is conjugation by $b$ on $\mathcal{B}^{\prime}$ and the identity on $\mathcal{B}^{\prime \prime}$. Then, $\Sigma\left(\alpha_{b} \alpha \overrightarrow{\mathcal{H}}\right)$ is obtained from $\Sigma\left(\alpha^{\prime} \overrightarrow{\mathcal{H}}^{\prime}\right) \vee I^{\prime} \vee I^{\prime \prime} \vee \Sigma\left(\alpha^{\prime \prime} \overrightarrow{\mathcal{H}}^{\prime \prime}\right)$ by collapsing $e$. We may continue until $I^{\prime \prime}$ and symmetrically $I^{\prime}$ are trivial. It follows from Lemma 9.21 that the result is a Gersten representative and that all Gersten representatives have this form.

\section{Proof of the Main Theorem}

Proposition 10.1 The algorithm of Section 2.10 is in fact an algorithm. 
Proof To detect a reduction, it is only necessary to be able to decide algorithmically if a homomorphism $\alpha: F_{\mathcal{B}} \rightarrow F_{\mathcal{B}^{\prime}}$ between free groups is an isomorphism. According to Stallings [27], $\alpha$ is injective if and only if the rank of the Stallings representative of $\alpha F(\mathcal{B})$ with respect to $\mathcal{B}^{\prime}$ is $|\mathcal{B}|$. It is surjective if and only if this Stallings representative is $R_{\mathcal{B}^{\prime}}$. Thus Step 1 is algorithmic.

Step 2 only depends on being able to find a Gersten representative and this is algorithmic by Corollary 9.15

After reducing and 1-simplifying, complexity has been reduced where complexity is the sequence of ranks of conjugacy classes of edge stabilizers viewed as an element of $\mathcal{L}$. Therefore this process stops.

We are finally in a position to prove Theorem 2.8 .

Proof of Theorem 2.8 By Proposition 10.1 we may assume that $\mathcal{G}$ is reduced. Let $S$ be the corresponding $G$-tree. We may also assume that no edge stabilizer is trivial of $S$. (Otherwise, $G$ has an obvious free decomposition and we may work with the factors instead of $G$.) If $G$ is freely decomposable, then by Lemma 8.5 there is a vertex $v \in V$ such that $\overrightarrow{\mathcal{H}}(v)$ can be 1 -simplified. In particular, by definition there is a basis $\mathcal{B}$ for $G_{v}$ with respect to which $\Sigma(\overrightarrow{\mathcal{H}}(v))$ may be visibly simplified. Since $\operatorname{Aut}\left(F_{\mathcal{B}}\right)$ acts transitively on bases, $\Sigma_{\mathrm{G}}(\overrightarrow{\mathcal{H}}(v))$ may be visibly simplified by Lemmas 9.19, 9.20, and 9.23.

We end with a few questions.

Question 1 Is there an algorithm to decide if the fundamental group of a finite graph of finite rank free groups is a surface group?

Question 2 Is there an algorithm to decide if the fundamental group of a finite graph of finite rank free groups splits over $\mathbb{Z}$ ?

Question 3 Is there an algorithm to find the $J S J$-decomposition of the fundamental group of a finite graph of finite rank free groups?

One can't hope to go too far in this direction since according to C. Miller 22 the isomorphism problem for finite graphs of finite rank free groups is unsolvable. 


\section{Appendix}

\section{A Bookkeeping}

In this section, details are provided as to how we record a finite graph of finite rank free groups $\mathcal{G}$ and how that data changes under a simplification. See Section 2.1 for notation.

A finite graph of finite rank free groups $\mathcal{G}$ is given by the following data:

- for each $e \in \hat{E}$, a basis $\mathcal{B}_{e}$ for $G_{e}$ such that $\mathcal{B}_{e}=\mathcal{B}_{e^{-1}}$;

- for $v \in V$, a basis $\mathcal{B}_{v}$ for $G_{v}$; and

- $\vec{w}=\left\{\vec{w}_{e}\right\}_{e \in \hat{E}}$ where $\vec{w}_{e}=\left\{w_{e, b}\right\}_{b \in \mathcal{B}_{e}}$ is a sequence of reduced words in $\mathcal{B}_{v}^{ \pm 1}$ representing $\left\{\varphi_{e}(b)\right\}_{b \in \mathcal{B}_{e}}$.

The Stallings algorithm referred to in Section 2.9 can be used to decide if a sequence $\vec{w}_{e}$ determines a monomorphism. Indeed, check if the rank of $\Sigma_{\mathrm{S}}$ obtained from $\vec{w}_{e}$ is equal to the rank of $G_{e}$.

Definition A.1 If $\left\{\vec{w}_{e}\right\}_{e \in \hat{E}}$ is a sequence as above, then we say another sequence $\left\{\vec{w}_{e}^{\text {out }}\right\}_{e \in \hat{E}}$ is conjugate to $\left\{\vec{w}_{e}\right\}_{e \in \hat{E}}$, written $\left\{\vec{w}_{e}^{\text {out }}\right\}_{e \in \hat{E}} \sim\left\{\vec{w}_{e}\right\}_{e \in \hat{E}}$, if there are $\left\{\psi_{e}=\psi_{e^{-1}} \in \operatorname{Aut}\left(G_{e}\right)\right\}_{e \in \hat{E}},\left\{\psi_{v} \in \operatorname{Aut}\left(G_{v}\right)\right\}_{v \in V}$, and $\vec{h}=\left\{h_{e} \in\right.$ $\left.G_{v}\right\}_{e \in \hat{E}}$ so that $\vec{w}_{e}^{\text {out }}$ represents $\left\{\psi_{v} \circ i_{h_{e}} \circ \varphi_{e} \circ \psi_{e}(b)\right\}_{b \in \mathcal{B}_{e}}$. If $\left\{\psi_{e}\right\}$ and $\left\{\psi_{v}\right\}$ are viewed as changing bases, then we see that $\left\{\vec{w}_{e}\right\}_{e \in \hat{E}}$ and $\left\{\vec{w}_{e}^{\text {out }}\right\}_{e \in \hat{E}}$ determine conjugate graphs of groups.

Definition A.2 For $e \in \hat{E}$ and $v=\partial_{0} e$, we say that given bases $\mathcal{B}_{e}$ and $\mathcal{B}_{v}$ are good if they determine decompositions of $G_{e}$ and $G_{v}$ that give a visual simplification. Specifically, we say that $\mathcal{B}_{v}$ and $\mathcal{B}_{e}$ are good in any of the following four cases.

blowing up There is a distinguished element $b_{v} \in \mathcal{B}_{v}$ so that $\varphi_{f}\left(\mathcal{B}_{f}\right) \subset\left\langle\hat{b}_{v}\right\rangle$, $f \in \hat{E}(v)$. There is no condition on $\mathcal{B}_{e}$ in this case. (We use the notation $\hat{b}_{v}=\mathcal{B}_{v} \backslash\left\{b_{v}\right\}$.)

unpulling There are distinguished elements $b_{v} \in \mathcal{B}_{v}$ and $b_{e} \in \mathcal{B}_{e}$ so that $\varphi_{e}\left(\hat{b}_{e}\right) \subset\left\langle\hat{b}_{v}\right\rangle, \varphi_{e}\left(b_{e}\right)=b_{v}$, and $\varphi_{f}\left(G_{f}\right) \subset\left\langle\hat{b}_{v}\right\rangle, f \in \hat{E}(v) \backslash\{e\}$.

unkilling There is a distinguished element $b_{v} \in \mathcal{B}_{v}$ and a partition $\mathcal{B}_{e}=$ $\mathcal{B}_{e}^{\prime} \sqcup \mathcal{B}_{e}^{\prime \prime}$ such that $\varphi_{e}\left(\mathcal{B}_{e}^{\prime}\right) \subset\left\langle\hat{b}_{v}\right\rangle, \varphi\left(\mathcal{B}_{e}^{\prime \prime}\right) \subset b_{v}\left\langle\hat{b}_{v}\right\rangle b_{v}^{-1}$, and $\varphi_{f}\left(G_{f}\right) \subset\left\langle\hat{b}_{v}\right\rangle$, $f \in \hat{E}(v) \backslash\{e\}$. 
cleaving There are partitions $\mathcal{B}_{v}=\mathcal{B}_{v}^{\prime} \sqcup \mathcal{B}_{v}^{\prime \prime}$ and $\mathcal{B}_{e}=\mathcal{B}_{e}^{\prime} \sqcup \mathcal{B}_{e}^{\prime \prime}$ such that $\varphi_{e}\left(\mathcal{B}_{e}^{\prime}\right) \subset\left\langle\mathcal{B}_{v}^{\prime}\right\rangle, \varphi\left(\mathcal{B}_{e}^{\prime \prime}\right) \subset\left\langle\mathcal{B}_{v}^{\prime \prime}\right\rangle$, and for $f \in \hat{E}(v) \backslash\{e\}$ either $\varphi_{f}\left(G_{f}\right) \subset$ $\left\langle\mathcal{B}_{v}^{\prime}\right\rangle$ or $\varphi_{f}\left(G_{f}\right) \subset\left\langle\mathcal{B}_{v}^{\prime \prime}\right\rangle$.

If $\mathcal{B}_{e}$ and $\mathcal{B}_{v}$ are good bases, then the corresponding simplification can be performed. The bases associated to edges and vertices of the simplified graphs are as follows. Unless explicitly mentioned, the words $w_{e, b}$ representing $\varphi_{e}(b)$ do not change. We use the notation as above and in Sections 2.3 2.6

blowing up After blowing up, $\mathcal{B}_{v}=\hat{b}_{v}$.

unpulling After unpulling, $\mathcal{B}_{e}=\hat{b}_{e}$ and $\mathcal{B}_{v}=\hat{b}_{v}$.

unkilling After unkilling, $\mathcal{B}_{e^{\prime}}=\mathcal{B}_{e}^{\prime}, \mathcal{B}_{e^{\prime \prime}}=\mathcal{B}_{e}^{\prime \prime}, \mathcal{B}_{v}=\hat{b}_{v}$, and $w_{e^{\prime \prime}, b}$ represents $b_{v}^{-1} \varphi_{e}(b) b_{v}$ for $b \in \mathcal{B}_{v}^{\prime \prime}$.

cleaving After cleaving, $\mathcal{B}_{v^{\prime}}=\mathcal{B}_{v}^{\prime}, \mathcal{B}_{v^{\prime \prime}}=\mathcal{B}_{v}^{\prime \prime}, \mathcal{B}_{e^{\prime}}=\mathcal{B}_{e}^{\prime}$, and $\mathcal{B}_{e^{\prime \prime}}=\mathcal{B}_{e}^{\prime \prime}$.

Proposition A.3 Let $\mathcal{G}$ be a finite graph of finite rank free groups given as in the beginning of this section. Suppose that, for some $v \in V, \Sigma_{\mathrm{G}}(\overrightarrow{\mathcal{H}}(v))$ can be visibly simplified. Then,

(1) there is $\mathcal{G}^{\text {out }} \sim \mathcal{G}$ such that $\mathcal{G}^{\text {out }}$ can be simplified; and

(2) If $\vec{w}$ specifies the bonding maps of $\mathcal{G}$, then a conjugate sequence $\vec{w}^{\text {out }}$ may be found algorithmically so that in $\mathcal{G}^{\text {out }}$ bases are good.

Proof Since (2) implies (1), it is enough to prove (2). The conjugate sequence $\vec{w}^{\text {out }}$ will be specified by supplying change of basis automorphisms $\left\{\psi_{f}=\psi_{f^{-1}} \in \operatorname{Aut}\left(G_{f}\right)\right\}_{f \in \hat{E}}$ and $\left\{\psi_{u} \in \operatorname{Aut}\left(G_{u}\right)\right\}_{u \in V}$ as well as the conjugating elements $\vec{h}=\left\{h_{f} \in G_{f}\right\}_{f \in \hat{E}}$ as in Definition A.1. The change of basis automorphisms can be given by specifying new bases.

Gersten's algorithm supplies $\alpha \in \operatorname{Aut}\left(G_{v}\right)$ so that $\operatorname{core}\left(\Sigma_{\mathrm{S}}(\alpha \vec{H}(v))\right)=\Sigma_{\mathrm{G}}(\overrightarrow{\mathcal{H}}(v))$. By taking $\psi_{v}=\alpha$, we obtain a conjugate sequence (still denoted $\vec{w}$ ) such that core $(\Sigma)$ can be visibly simplified where $\Sigma=\Sigma_{\mathrm{S}}(\vec{H}(v))$. We are using the convention that all unmentioned change of basis and conjugating automorphisms are identities.

Recall that each component of $\Sigma$ has a basepoint. Choose shortest paths in each component of $\Sigma$ from the basepoint to the core and take as conjugating elements the words read off along the inverses of these paths. The resulting conjugate sequence has basepoints in core $(\Sigma)$. Hence, we may further assume that $\Sigma=\operatorname{core}(\Sigma)$. 
If $\Sigma$ can be visibly blown up, then bases are good and if $b \in \mathcal{B}_{v}$ is an element that does not appear as a label on $\Sigma$ we take $b$ for the distinguished element $b_{v}$.

In each of the remaining cases, there is a distinguished $e \in \hat{E}(v)$. Let $\Sigma_{e}$ be the component of $\Sigma$ indexed by $e$, ie, $\Sigma_{e}=\Sigma_{\mathrm{S}}\left(\varphi_{e}\left(G_{e}\right)\right)$. There is the natural factorization $G_{e} \rightarrow \pi_{1}\left(\Sigma_{e}, *\right) \rightarrow G_{v}$ of $\varphi_{e}$ where the first map is the isomorphism coming from Stallings algorithm (see Section 2.9) and the second map is induced by natural map of Section 2.8. We will use this first map to identify $G_{e}$ with $\pi_{1}\left(\Sigma_{e}, *\right)$. If a basis for $\pi_{1}\left(\Sigma_{e}, *\right)$ is given via a maximal tree $T$ for $\Sigma_{e}$, it is easy to write this isomorphism in terms of the given bases. To make this identification explicit, it is necessary to invert the automorphism and this can be done algorithmically, see Remark 9.8. Via our identification, $T$ determines a new basis for $G_{e}$ and hence a conjugate sequence.

If $\Sigma$ can be visibly unpulled, then choose an edge $c$ of $\Sigma_{e}$ that does not separate its component and whose label $b \in \mathcal{B}_{v}$ appears exactly once in $\Sigma$. Choose a maximal tree $T$ for $\Sigma_{e}$ so that $c \not \subset T$ and let $\mathcal{B}_{e}^{\text {out }}$ be the basis determined by $T$. There is an element $b_{e} \in \mathcal{B}_{e}^{\text {out }}$ corresponding to $c$. Let $b_{v}$ be $\varphi_{e}$-image in $G_{v}$ of $b_{e}$. Set $\mathcal{B}_{v}^{\text {out }}=\left\{b_{v}\right\} \sqcup \hat{b}$ which is a basis for $G_{v}$ since $b$ appears exactly once in $b_{v}$ when expressed as a $\mathcal{B}_{v}$-word. The new bases are good and determine change of basis automorphisms giving rise to the desired $\vec{w}^{\text {out }}$.

If $\Sigma$ can be visibly unkilled, then choose an edge $c$ of $\Sigma_{e}$ that separates its component and whose label $b \in \mathcal{B}_{v}$ appears exactly once in $\Sigma$. By changing the orientation of $c$ and inverting $b$ if necessary, we may assume that $\partial_{0} c$ and the basepoint are in the same component of the graph obtained by removing $c$ from $\Sigma_{e}$. Let $T$ be a maximal tree for $\Sigma_{e}$. This gives the desired new basis $\mathcal{B}_{e}^{\text {out }}$. Choosing a shortest path in $T$ from the $\partial_{0} c$ to the basepoint gives rise to a conjugating element $h_{e}$ which has the effect of changing the basepoint of $\Sigma_{e}$ to $\partial_{0} c$. The new bases are good. Indeed, the partition of $\mathcal{B}_{e}^{\text {out }}$ is induced by the separating edge. More precisely, let $\left(\mathcal{B}_{e}^{\text {out }}\right)^{\prime \prime}$ be the elements of $\mathcal{B}_{e}^{\text {out }}$ that contain the letter $b$ and let $\left(\mathcal{B}_{e}^{\text {out }}\right)^{\prime}$ be the complement. Set $b_{v}=b$.

If $\Sigma$ can be visibly cleaved, then write $\Sigma=\Sigma^{\prime} \vee \Sigma^{\prime \prime}$ respecting the non-trivial partition $\mathcal{B}_{v}^{\prime} \sqcup \mathcal{B}_{v}^{\prime \prime}$ of $\mathcal{B}_{v}$. A choice of maximal tree $T$ for $\Sigma_{e}$ gives the desired new basis $\mathcal{B}_{e}^{\text {out }}$ and a shortest path from the wedge point to the basepoint determines a conjugating element $h_{e}$. With these choices, the new bases are good. Indeed, the partition of $\mathcal{B}_{e}^{\text {out }}$ is induced by the wedge. More precisely, $\left(\mathcal{B}_{e}^{\text {out }}\right)^{\prime}$ corresponds to the set of edges of $\Sigma^{\prime} \cap \Sigma_{e}$ not in $T$ and $\left(\mathcal{B}_{e}^{\text {out }}\right)^{\prime \prime}$ corresponds to the remaining edges not in $T$. 
Example A.4 Let $\mathcal{G}$ have underlying graph $\Gamma$ as in Figure 16. Suppose that $\mathcal{B}_{e}=\left\{a_{1}, a_{2}\right\}, \mathcal{B}_{v}=\left\{b_{1}, b_{2}\right\}$, and $\mathcal{B}_{f}=\{z\}$. We will not specify $G_{u}$ since it will not change. Suppose that $\varphi_{e}\left(a_{1}\right)=b_{1}^{2} b_{2}^{2}, \varphi_{e}\left(a_{2}\right)=b_{1}^{2} b_{2}^{2} b_{1}^{2}, \varphi_{f}(z)=$ $b_{1}, \varphi_{f^{-1}}(z)=b_{2}$. Then, $\Sigma(v)=\Sigma_{\mathrm{S}}(\vec{H}(v))$ is a Gersten representative, ie, $\Sigma_{\mathrm{S}}(\vec{H}(v))=\Sigma_{\mathrm{G}}(\overrightarrow{\mathcal{H}}(v)) . \quad \Sigma(v)$ is displayed in Figure 16, and it can be visibly cleaved. The given bases are not good. A good basis for $G_{e}$ corresponds to the one determined by the wedge point in $\Sigma(v)$. The change of basis automorphism $\psi_{e} \in \operatorname{Aut}\left(G_{e}\right)$ is given by $\psi_{e}\left(a_{1}\right)=a_{1}^{-1} a_{2}$ and $\psi_{e}\left(a_{2}\right)=a_{2}^{-1} a_{1} a_{1}$, ie, $\varphi_{e} \circ$ $\psi_{e}\left(a_{1}\right)=b_{1}^{2}$ and $\varphi_{e} \circ \psi_{e}\left(a_{2}\right)=b_{2}^{2}$.

If $\mathcal{G}^{\prime}$ is the result of cleaving $\mathcal{G}$, then $\mathcal{B}_{e^{\prime}}=\left\{a_{1}\right\}, \mathcal{B}_{e^{\prime \prime}}=\left\{a_{2}\right\}, \mathcal{B}_{v^{\prime}}=\left\{b_{1}\right\}$, $\mathcal{B}_{v^{\prime \prime}}=\left\{b_{2}\right\}, \varphi_{e^{\prime}}\left(a_{1}\right)=b_{1}^{2}, \varphi_{e^{\prime \prime}}\left(a_{2}\right)=b_{2}^{2}, \varphi_{\left(e^{\prime}\right)^{-1}}\left(a_{1}\right)=\varphi_{e^{-1}} \circ \psi_{e}\left(a_{1}\right)$, and $\varphi_{\left(e^{\prime \prime}\right)^{-1}}\left(a_{2}\right)=\varphi_{e^{-1}} \circ \psi_{e}\left(a_{2}\right)$.

The next step in the algorithm would be to reduce $\mathcal{G}^{\prime}$. The example could have been complicated by post-composing $\varphi_{e}, \varphi_{f}$, and $\varphi_{f^{-1}}$ by some $\psi_{v} \in \operatorname{Aut}\left(G_{v}\right)$. In that case, we would use Gersten's algorithm first (and discover $\psi_{v}$ ).

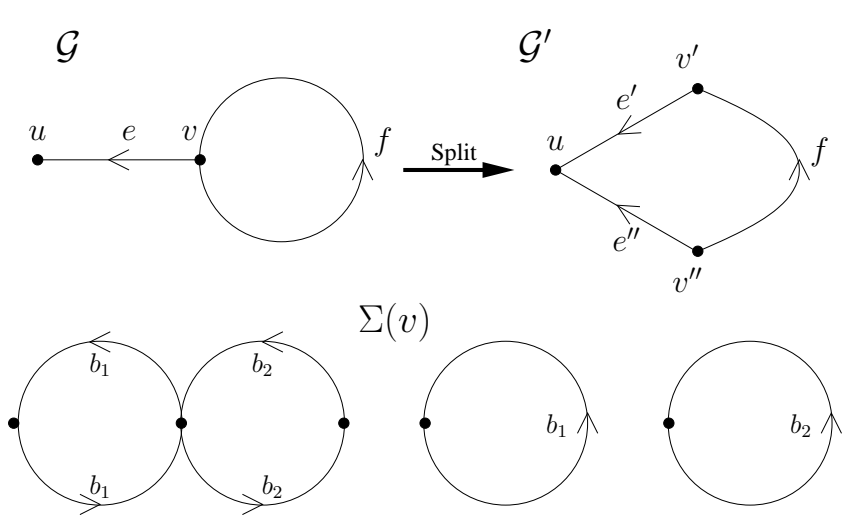

Figure 16

\section{References}

[1] H Bass, Some remarks on group actions on trees, Comm. Algebra 4 (1976) 1091-1126 MathReview

[2] M Bestvina, M Feighn, Outer limits, preprint (1994)

[3] M Bestvina, M Feighn, A combination theorem for negatively curved groups, J. Differential Geom. 35 (1992) 85-101 MathReview 
The Grushko decomposition of a finite graph of free groups

[4] M Bestvina, M Feighn, Bounding the complexity of simplicial group actions on trees, Invent. Math. 103 (1991) 449-469 MathReview

[5] MR Bridson, A Haefliger, Metric spaces of non-positive curvature, Grundlehren series 319, Springer-Verlag, Berlin (1999) MathReview

[6] MR Bridson, D T Wise, $\mathcal{V H}$ complexes, towers and subgroups of $F \times F$, Math. Proc. Cambridge Philos. Soc. 126 (1999) 481-497 MathReview

[7] P Brinkmann, Splittings of mapping tori of free group automorphisms, Geom. Dedicata 93 (2002) 191-203 MathReview

[8] D E Cohen, Combinatorial group theory: a topological approach, London Mathematical Society Student Texts 14, Cambridge University Press, Cambridge (1989) MathReview

[9] G-A Diao, Is a graph of finitely generated free groups free? An algorithm, $\mathrm{PhD}$ thesis, Rutgers University, Newark (2003)

[10] M Feighn, M Handel, Mapping tori of free group automorphisms are coherent, Ann. of Math. (2) 149 (1999) 1061-1077 MathReview

[11] K Fujiwara, P Papasoglu, JSJ-decompositions of finitely presented groups and complexes of groups, to appear in GAFA

[12] R Geoghegan, M L Mihalik, M Sapir, D T Wise, Ascending HNN extensions of finitely generated free groups are Hopfian, Bull. London Math. Soc. 33 (2001) 292-298 MathReview

[13] V Gerasimov, Detecting connectedness of the boundary of a hyperbolic group, preprint (1999)

[14] S M Gersten, On Whitehead's algorithm, Bull. Amer. Math. Soc. (N.S.) 10 (1984) 281-284 MathReview

[15] IA Grushko, On generators of a free product of groups, Matem. Sbornik N. S. 8 (1940) 169-182

[16] A Hatcher, Algebraic topology, Cambridge University Press, Cambridge (2002) MathReview

[17] W Jaco, D Letscher, J H Rubinstein, Algorithms for essential surfaces in 3-manifolds, from: "Topology and geometry: commemorating SISTAG", Contemp. Math. 314, Amer. Math. Soc., Providence, RI (2002) 107-124 MathReview

[18] S Kalajdžievski, Automorphism group of a free group: centralizers and stabilizers, J. Algebra 150 (1992) 435-502 MathReview

[19] O Kharlampovich, A Myasnikov, Irreducible affine varieties over a free group. I. Irreducibility of quadratic equations and Nullstellensatz, J. Algebra 200 (1998) 472-516 MathReview

[20] O Kharlampovich, A Myasnikov, Effective JSJ decompositions, from: "Groups, languages, algorithms", (Borovik, editor), Contemp. Math. 378, Amer. Math. Soc., Providence, RI (2005) 87-212 
[21] R C Lyndon, P E Schupp, Combinatorial group theory, Classics in Mathematics, Springer-Verlag, Berlin (2001) MathReview

[22] C F Miller, III, On group-theoretic decision problems and their classification, Princeton University Press, Princeton, N.J. (1971) MathReview

[23] E Rips, Z Sela, Cyclic splittings of finitely presented groups and the canonical JSJ decomposition, Ann. of Math. (2) 146 (1997) 53-109 MathReview

[24] Z Sela, Diophantine geometry over groups. I. Makanin-Razborov diagrams, Publ. Math. Inst. Hautes Études Sci. (2001) 31-105 MathReview

[25] J-P Serre, Trees, Springer Monographs in Mathematics, Springer-Verlag, Berlin (2003) MathReview

[26] A Shenitzer, Decomposition of a group with a single defining relation into a free product, Proc. Amer. Math. Soc. 6 (1955) 273-279 MathReview

[27] J R Stallings, Topology of finite graphs, Invent. Math. 71 (1983) 551-565 MathReview

[28] J R Stallings, Foldings of G-trees, from: "Arboreal group theory (Berkeley, CA, 1988)", Math. Sci. Res. Inst. Publ. 19, Springer, New York (1991) 355-368 MathReview

[29] G A Swarup, Decompositions of free groups, J. Pure Appl. Algebra 40 (1986) 99-102 MathReview

[30] J H C Whitehead, On certain sets of elements in a free group, Proc. London Math. Soc. 41 (1936) 48-56

[31] J H C Whitehead, On equivalent sets of elements in a free group, Ann. of Math. (2) 37 (1936) 782-800 MathReview 